\title{
Juckreizmanagement im Kindesalter
}

\author{
Regina Fölster-Holst
}

Klinik für Dermatologie, Venerologie und Allergologie, Universitätsklinikum Schleswig-Holstein, Campus Kiel, Kiel, Deutschland

\section{Zusammenfassung}

Juckreiz ist bei Kindern ein sehr verbreitetes Symptom und steht in der Regel eher mit einer Hautkrankheit in Verbindung als mit einer zugrunde liegenden systemischen Erkrankung. Zu den häufigsten Dermatosen zählen atopische Dermatitis, Kontaktdermatitis, Insektenstiche, Skabies und Pediculosis capitis. Es gibt spezifische diagnostische Muster, die sich aus einer sorgfältigen Erhebung der Vor- geschichte und der dermatologischen Untersuchung ergeben. Bei der dermatologischen Behandlung muss berücksichtigt werden, dass Kinder, insbesondere im frühen Kindesalter, im Vergleich zu Erwachsenen Unterschiede in der Physiologie und Pathophysiologie sowie in der Pharmakokinetik und Pharmakodynamik aufweisen.

(c) 2017 S. Karger GmbH, Freiburg
Juckreiz (Pruritus) ist ein verbreitetes Symptom bei Kindern. Insofern ist es erstaunlich, dass es keine Daten zur Prävalenz von Juckreiz im Kindesalter bezogen auf die Allgemeinbevölkerung gibt. Die Suche nach «Juckreiz UND Kinder» und «Pruritus UND Kinder» in PubMed (bis einschließlich 9. März 2016) ergab 2629 bzw. 2136 Veröffentlichungen, die sich jedoch in der Mehrzahl auf bestimmte Erkrankungen bezogen und meist Fallberichte beinhalteten. Pruritus im Kindesalter ist hauptsächlich mit Dermatosen assoziiert. Im Vergleich zu Erwachsenen sind systemische Erkrankungen und Arzneimittelreaktionen selten. Zu den juckenden Dermatosen im Kindesalter zählen ekzematöse Erkrankungen (insbesondere die atopische Dermatitis), Exantheme, Infektionen/ Infestationen, Urtikaria/Mastozytose, Autoimmunerkrankungen sowie Genodermatosen. Bei der dermatologischen Behandlung (topisch und systemisch) müssen Besonderheiten im Kindesalter hinsichtlich der Physiologie und Pathophysiologie sowie der Pharmakokinetik und Pharmakodynamik berücksichtigt werden, die sich von den Merkmalen bei Erwachsenen unterscheiden.

\section{Besonderheiten der Hautphysiologie, Pathophysiologie und Hautpflege im frühen Kindesalter, die bei der Behandlung zu berücksichtigen sind}

Im Kindesalter, besonders im frühen Kindesalter sind Besonderheiten hinsichtlich der Hautphysiologie und der Pathophysiologie zu berücksichtigen. Die Resorption von topisch applizierten Wirkstoffen und Emollientien (Pflegeprodukte) wird durch das hohe Verhältnis von Körperoberfläche zu Körpergewicht [1] bestimmt.
Im Kindesalter ist die Haut darüber hinaus durch eine dünnere Epidermis und eine dünnere Hornschicht (Stratum corneum) sowie durch kleinere Korneozyten gekennzeichnet $[1,2]$. Physikalische Messungen der epidermalen Barrierefunktion zeigen, dass natürliche Feuchtigkeitsfaktoren und Oberflächenlipide bei der Haut von Kleinkindern in geringeren Konzentrationen vorhanden sind als bei Erwachsenen. Außerdem liegen ein hoher pH-Wert, eine hohe Desquamation (Hornhautabschuppung), hohe Proliferationsraten und ein höherer transepidermaler Wasserverlust vor [3]. Diese Eigenschaften führen zu einer beeinträchtigten Epidermisstruktur und -funktion, was zu einer hohen Resorption topisch applizierter Wirkstoffe und einer hohen Penetration durch Umweltfaktoren wie Reizstoffe, Allergene und Infektionserreger führt [4]. Diese Tatsachen sollten generell bei der Behandlung von Kindern beachtet werden, das gilt insbesondere für Kinder, die an Krankheiten mit einem bekannten Barrieredefekt wie der atopischen Dermatitis leiden [5]. Neben den Eigenschaften der Hautbarriere hängt die perkutane Resorption von Arzneimitteln und von Pflegeprodukten jedoch auch von den physikalischen und chemischen Eigenschaften des Arzneimittels bzw. Pflegeproduktes ab [6]. Im frühen Kindesalter angewendete Emollientien sollten frei von Duft-, Farb- und Konservierungsstoffen sein, da diese bekanntermaßen mit dem Risiko einer Reizung oder Allergenität verbunden sind. Auch wenn Emollientien nicht per se zu den antipruritogenen Externa gezählt werden, fetten und befeuchten sie die Haut, sie schützen die Integrität der Hornschicht und der Hautbarriere und dienen der Behandlung trockener Haut [7]. Das kann durchaus mit einer Linderung des Juckreizes verbunden sein $[8,9]$.

\section{KARGER \\ Fax +497614520714 \\ information@karger.com}

www.karger.com
() 2017 S. Karger GmbH, Freiburg

Accessible online at:

www.karger.com/kkd
Prof. Dr. Regina Fölster-Holst

Klinik für Dermatologie, Venerologie und Allergologie,

Universitätsklinikum Schleswig-Holstein, Campus Kiel

Arnold-Heller-Straße 3, Haus 19, 24105 Kiel, Deutschland

rfoelsterholst@dermatology.uni-kiel.de 
Tab. 1. Juckende ekzematöse Krankheiten (Auswahl)

\begin{tabular}{|c|c|c|}
\hline Krankheiten & Klinisches Muster & Alter bei Krankheitsbeginn \\
\hline \multicolumn{3}{|l|}{ Dermatitis } \\
\hline Atopische Dermatitis & $\begin{array}{l}\text { Ekzem der Extremitäten, Hautfalten; mit Asthma, } \\
\text { allergischer Rhinitis und Nahrungsmittelallergie } \\
\text { assoziiert }\end{array}$ & $\begin{array}{l}\text { vor allem im ersten Lebens- } \\
\text { jahr }\end{array}$ \\
\hline Kontaktdermatitis & Ekzeme an den Kontaktstellen & jederzeit \\
\hline $\begin{array}{l}\text { Seborrhoische Dermatitis im } \\
\text { Kleinkindalter }\end{array}$ & Ekzem an Achseln, Leisten und im Windelbereich & in den ersten Lebenswochen \\
\hline \multicolumn{3}{|l|}{ Infestationen } \\
\hline Skabies (infantile) & $\begin{array}{l}\text { Gänge, verbreitetes Ekzem (einschließlich Gesicht } \\
\text { und Kopf), Pusteln an Fußsohlen }\end{array}$ & jederzeit \\
\hline Pediculosis capitis & Papeln, Pusteln, Ekzeme an Kopf und Hals & vor allem im Schulalter \\
\hline \multicolumn{3}{|l|}{ Genodermatosen } \\
\hline Omenn-Syndrom & $\begin{array}{l}\text { Quintett aus Ekzem, Haarschaftanomalie, } \\
\text { Alopezie, Durchfall und Infektionen }\end{array}$ & bei der Geburt \\
\hline DiGeorge-Syndrom & $\begin{array}{l}\text { Ekzem, Herzanomalien, Gaumenspalte, } \\
\text { Thymusaplasie oder -hypoplasie }\end{array}$ & bei Neugeborenen \\
\hline Wiskott-Aldrich-Syndrom & Ekzem, Petechien, Infektionen & bei der Geburt \\
\hline Netherton-Syndrom & Ekzem, Atopie, Hautinfektionen & bei der Geburt \\
\hline Akrodermatitis enteropathica & $\begin{array}{l}\text { Trias aus ekzematösen Läsionen (periorifiziell, } \\
\text { Finger und Zehen), Alopezie und Durchfall }\end{array}$ & $\begin{array}{l}2 \text { Wochen nach dem } \\
\text { Abstillen }\end{array}$ \\
\hline $\begin{array}{l}\text { IPEX-Syndrom (Immundysregulation, } \\
\text { Polyendokrinopathie, Enteropathie, } \\
\text { mit X-Chromosom verknüpft) }\end{array}$ & $\begin{array}{l}\text { Ekzem, Atopie, Diabetes mellitus, Enteropathie, } \\
\text { Nahrungsmittelallergie }\end{array}$ & erste Lebensmonate \\
\hline \multicolumn{3}{|l|}{ Neoplasie } \\
\hline $\begin{array}{l}\text { Langerhans-Zell-Histiozytose im } \\
\text { Kleinkindalter }\end{array}$ & $\begin{array}{l}\text { bräunliche Papeln, Papulovesikel, impetigoartige } \\
\text { Läsionen der Achselhöhlen und Leisten, } \\
\text { seborrhoische Läsionen auf der Kopfhaut }\end{array}$ & $\begin{array}{l}\text { bei der Geburt oder in den } \\
\text { ersten } 2 \text { Jahren }\end{array}$ \\
\hline
\end{tabular}

\section{Besonderheiten der Vorgeschichte, der dermatologischen} Untersuchung und der Diagnosen im Kindesalter

Die medizinische Versorgung ist im Kindesalter in vielerlei Hinsicht anders als im Erwachsenenalter. Um brauchbare Aussagen während der Anamnese zu erhalten, ist ein angemessener Kontakt sowohl mit dem Kind als auch mit den Eltern notwendig. Wenn die Kinder im Vorschul-/Schulalter sind, sollten die Fragen - auch hinsichtlich des Juckreizes - sowohl an die Eltern als auch an das Kind gerichtet werden. Der Dialog zwischen Arzt und Patient ist die Basis für ein gutes Vertrauensverhältnis [4]. Dies ist auch für die Therapietreue von Bedeutung [4].

In der Anamnese unterscheiden sich die Fragen zum Juckreiz bei Kindern nicht so sehr von denen bei Erwachsenen. Allerdings sind in dieser Lebensphase besondere Probleme zu berücksichtigen, darunter der Kontakt mit anderen Kindern, die Kleidung, Impfungen im Rahmen der regelmäßigen pädiatrischen Kontrolluntersuchungen, alltägliche Aktivitäten und Essgewohnheiten. Bei der Aufnahme der Anamnese zum Juckreiz im Kindesalter sind die folgenden Fragen und Untersuchungen hilfreich:
- Ist der Juckreiz lokalisiert (regionale Infektion, lokalisierte Dermatose) oder generalisiert (symmetrische Verteilung bei generalisierten Dermatosen oder systemischen Erkrankungen)? - Leiden auch andere Familienmitglieder unter Juckreiz?

- Gibt es eine Vorgeschichte von bekannten Krankheiten und/ oder Allergien des Kindes und/oder anderer Familienmitglieder (z.B. atopische Krankheiten)?

- Wann hat der Juckreiz begonnen und wie ist die Qualität des Juckreizes?

- Hält die Familie Haustiere im Haus? In welcher Umgebung lebt das Kind?

- Ist das Kind in einem guten Allgemeinzustand?

- Gibt es Lebensmittel, die bei dem Kind Juckreiz auslösen?

- Leidet das Kind unter einer Infektion, die mit Juckreiz verbunden ist?

- Gibt es emotionale Belastungen oder psychiatrische Erkrankungen in der Vorgeschichte?

- Welche Produkte verwenden das Kind/die Eltern für die Hautpflege? Wie sind die Badegewohnheiten des Kindes (Häufigkeit pro Woche, Temperatur, Dauer usw.)?

- Nimmt das Kind Medikamente? Wenn ja, welche Medikamente, für welche Indikation und wie lange schon? 
Abb. 1. Nummuläre Form der atopischen Dermatitis, mit sehr starken Absonderungen.

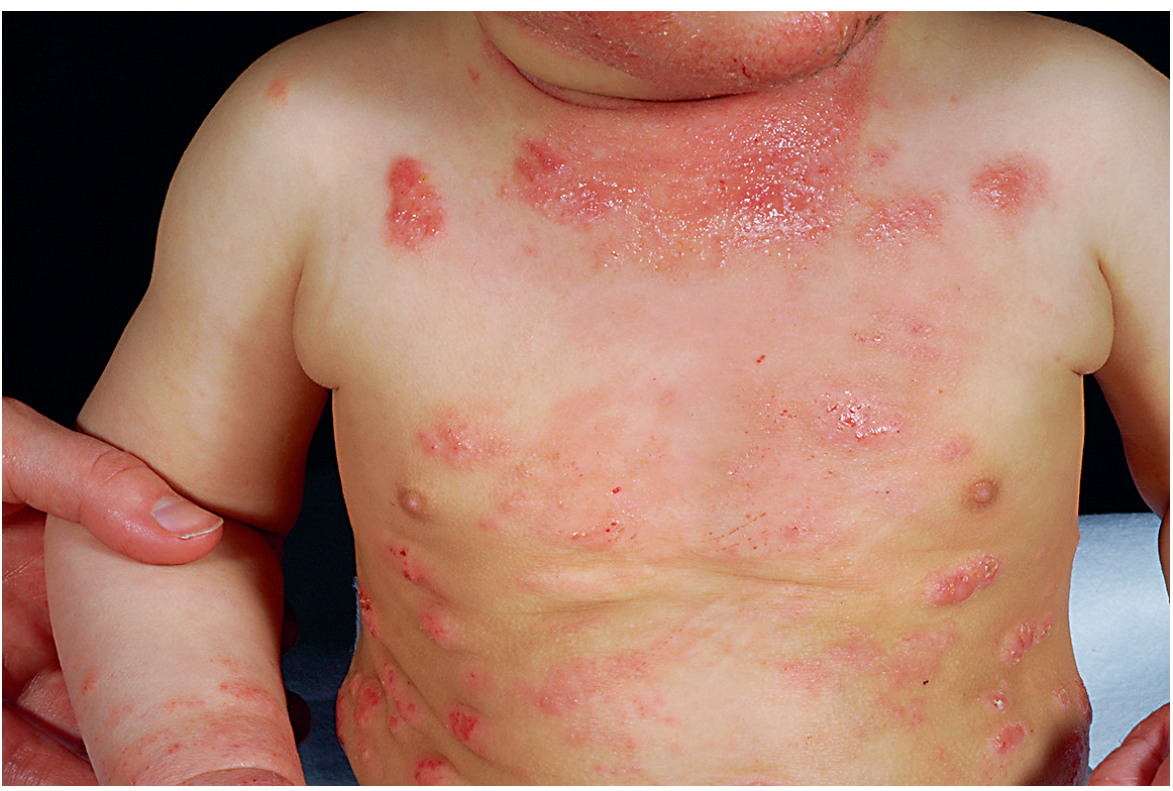

- Welche Kleidung trägt das Kind (Baumwolle, Seide, Wolle, andere)?

- Gibt es Infektionen im Umfeld (Kindergarten, Schule usw.)?

Die klinische Untersuchung eines Kindes unterscheidet sich von der bei Erwachsenen. Dazu gehört nicht nur eine Kommunikation auf dem Niveau des Kindes, was unter anderem eine andere Ansprache erfordert, sondern auch die Beurteilung des Juckreizes durch das Kind. In der Literatur gibt es einige Bewertungsskalen für Juckreiz, die im Kindesalter verwendet werden können, beispielsweise die Juckreiz-Bewertungsskala für den «Pediatric Burn Survivor» [10]. Diese «Itch-Man-Scale» korreliert mit anderen Skalen, z.B. der 5D-Juckreiz-Skala und der visuellen Analogskala. Darüber hinaus ist zu beachten, dass es Krankheiten gibt, die nur oder zumindest überwiegend im Kindesalter auftreten. Zu diesen Krankheiten gehören die infantile eosinophile pustulöse Follikulitis, die infantile Akropustulose, das Gianotti-Crosti-Syndrom, Varizellen (Windpocken) und Neurofibromatose 1. Diese und andere Krankheiten werden in den folgenden Abschnitten betrachtet.

\section{Ekzematöse Erkrankungen}

Ekzematöse Erkrankungen im Kindesalter sind von Juckreiz begleitet und umfassen die atopische Dermatitis, seborrhoische Dermatitis (weniger Juckreiz), Kontaktdermatitis und Genodermatosen. Darüber hinaus ist zu berücksichtigen, dass es viele kutane Infektionen und Infestationen gibt, die im Verlauf der Krankheit typischerweise ekzematöse Läsionen aufweisen, wie Impetigo, Skabies (Krätze) oder Pediculosis capitis (Kopflausbefall). Die charakteristischen Merkmale der verschiedenen ekzematösen Krankheiten werden jeweils im Detail behandelt, da sie unterschiedliche Managementstrategien erfordern.

\section{Atopische Dermatitis}

Die atopische Dermatitis ist eine chronische, rezidivierende entzündliche Dermatose, deren Hauptmerkmal intensiver Juckreiz ist. Dieser ist mit Schlaf- und Konzentrationsstörungen verbunden und darüber hinaus mit sozialer Stigmatisierung [11]. In den ersten Lebenstagen ist Pruritus manchmal schwer zu erkennen. Verglichen mit älteren Kindern und Erwachsenen zeigen Säuglinge andere Prädilektionsstellen und Morphologien. Zuerst sind Kopf und Gesicht betroffen, gefolgt von den Extremitätenstreckseiten [4]. Diese Läsionen zeichnen sich häufig durch Erythem, Papeln, Pusteln, Krusten und Nässen aus.

Die typische Lichenifikation in den Falten der Extremitäten, die vermutlich mit dem permanenten Kratzen zusammenhängen, sind in der späteren Kindheit zu beobachten. Eine sehr spezielle, im Kindesalter häufig auftretende Form ist das nummuläre Ekzem, das eine starke Entzündung aufweist (Abb. 1) und, im Vergleich zu Erwachsenen, neben den Extremitäten auch den Rumpf betrifft. Viele Kinder zeigen ein sehr frühes Auftreten der Erkrankung, und die atopische Dermatitis kann sich als Erythrodermie mit feiner Hautschuppung manifestieren. In diesen Fällen sollten verschiedene Differenzialdiagnosen in Betracht gezogen werden (Tab. 1). Es empfiehlt sich, bei jedem erythrodermischen Neugeborenen einige Haare (Kopfhaar, Wimpern oder Augenbrauen) für die mikroskopische Analyse des Haarschaftes abzuschneiden, um das Netherton-Syndrom mit dem krankheitstypischen Merkmal der Trichorrhexis invaginata (Bambushaare) auszuschließen. Die Diagnose kann durch eine molekulare Analyse überprüft werden, die die Mutation des SPINK5-Gens nachweist, das für LEKTI, einen Serinprotease-Inhibitor, kodiert [12]. Das Netherton-Syndrom hat viele Symptome mit der atopischen Dermatitis und anderen atopischen Erkrankungen gemeinsam, unter anderem starken Pruritus [4, 13]. Dies gilt auch für das generalisierte Peeling-Skin-Syndrom (Abb. 2), das mit Mutationen im $C D S N-G e n$ zusammenhängt. Dies führt zu einem vollständigen Verlust von Corneodesmosin, was wiederum mit einem schweren Defekt der epidermalen Barriere verbunden ist. Klinisch sind Erythrodermie, generalisierte Hautabschuppung und unerträglicher Juckreiz die typischen Merkmale [14]. 


\section{2) Stelara:}

\section{Stark auf}

\section{ganzer
Strecke. \\ Strecke.}

\section{Gibt alles!}

Bei mittelschwerer bis schwerer Plaque-Psoriasis.*
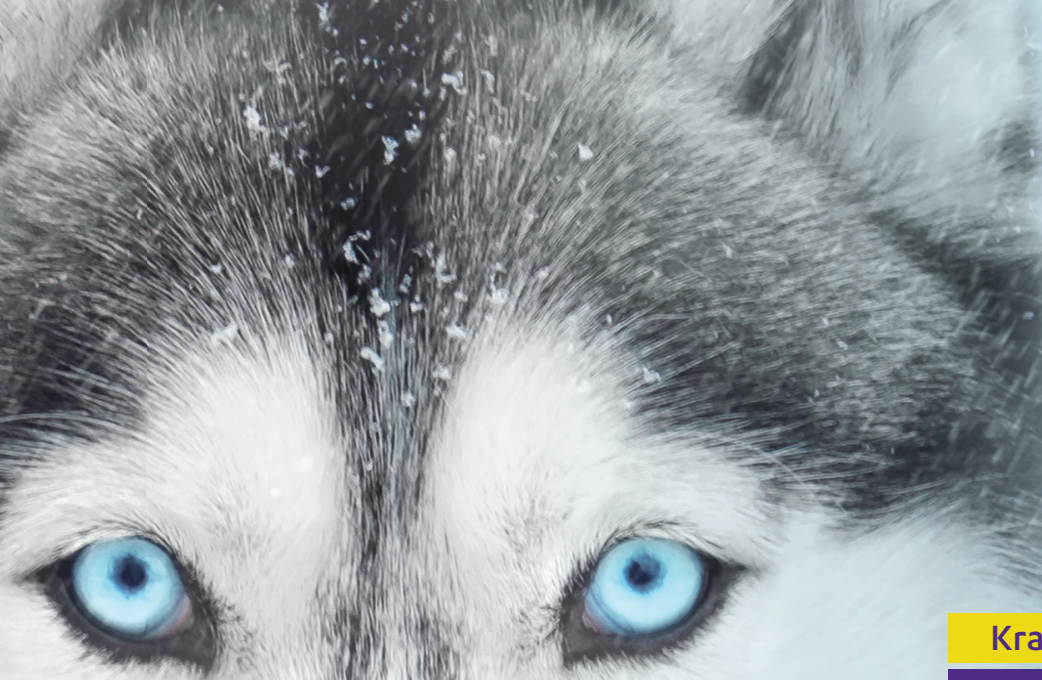

Kraft.

Ausdauer.

*STELARA" ist für die Behandlung erwachsener Patienten mit mittelschwerer bis schwerer Plaque-Psoriasis indiziert, bei denen andere systemische Therapien einschließlich Ciclosporin, Methotrexat (MTX) oder PUVA (Psoralen und Ultraviolett A) nicht angesprochen haben, kontraindiziert sind oder nicht vertragen wurden.

STELARA 45 mg Injektionslösung, STELARA 45 mg/- 90 mg Injektionslösung in einer Fertigspritze, STELARA* $130 \mathrm{mg}$ Konzentrat zur Herstellung einer Infusionslösung. Wirkstoff: Ustekinumab. Zusammensetz.: 1 Durchstechflasche enth. $45 \mathrm{mg}$ Ustekinumab in 0,5 ml bzw. $130 \mathrm{mg}$ Ustekinumab in $26 \mathrm{ml}$. 1 Fertigspritze enth. $45 \mathrm{mg}$ Ustekinumab in 0,5 ml bzw. $90 \mathrm{mg}$ Ustekinumab in 1,0 ml. Sonst. Bestandt.: $45 \mathrm{mg}$ Durchstechlasche bzw. $45 \mathrm{mg} /-90 \mathrm{mg}$ Fertigspritze: Histidin, Histidinhydrochlorid-Monohydrat, Polysorbat 80, Sucrose, Wasser f. InjektionsMonohydrat, Methionin, Polysorbat 80, Sucrose, Wasser für Injektionszw.. Anw.geb.: STELARA ist für $d$. Bhdlg. erw. Pat. mit mittelschwerer bis schwerer Plaque-Psoriasis indiziert, bei denen and. systemische Therapien einschl. Ciclosporin, Methotrexat od. PUVA nicht angesprochen haben, kontraindiziert sind od. nicht vertragen wurden. STELARA ist für $\mathrm{d}$. Bhdlg. mittelschwere bis schwerer Plaque-Psoriasis b. Kindern u. Jugendl. ab 12 Jahren indiziert, d. unzureich. auf and. systemische Therapien od. Phototherapien angesprochen od. sie nicht vertragen haben.

STELARA ist allein od. in Kombin. m. Methotrexat für d. Bhdlg. d. aktiven psoriat. Arthritis b. erw. Pat indiziert, wenn d. Ansprechen aufe vorherige nicht-biolog krankheitsmodifiz antirheumat. (DMARD) Therapie unzureich ewesen ist STELARA* ist für d Bhdl rem bis schwerem aktiven Morbus Crohn indiziert, die entweder auf eine konventionelle Therapie od. einen d. Tumornekrosefaktor-alpha (TNF $\alpha$ )-Antagonisten unzureich. angesprochen haben, nicht mehr darauf ansprechen od. eine Unverträglichk. od. eine Kontraindik. gg. eine entsprech. Bhdlg. aufweisen. Gegenanz.: Überempfindl. gg. Ustekinumab od. and. Bestandt.; klin. relevante aktive Infektion (z. B. aktive Tuberkulose); Pat. unter 18 Jahren (Morbus Crohn u. psoriat. Arthritis) bzw. unter 12 Jahren (Plaque-Psoriasis); Schwangerschaft; Stillzeit (b. Entschdg. üb. Aussetzen d. Stillens währ. d. Bhdlg. u. bis zu 15 Wo. danach od. Aussetzen d. Therapie m. STELARA ${ }^{*}$ muss Nutzen $d$. Stillens für $d$. Kind $m$. Nutzen $d$. Therapie für $d$. Frau abgewogen werden). Vorsicht bei: Pat. mit chron. Infekt. od. einer rezidiv. Infektion in d. Anamnese. Immunsuppressiva wie STELARA können d. Risiko v. malignen Tumoren u. d. Risiko v. Infektionen erhöhen u. latente Infektionen reaktivieren. Pat. mit malignen Tumoren (auch in d. Vorgeschichte). Bhdlg. v. latenter Tuberkulose muss vor Verabreichg. v. STELARA" eingeleitet werden.
Anti-Tuberkulose-Bhdlg. soll vor Verabr. v. STELARA" b. Pat. m. Anamnese v. latenter od. aktiv. Tuberkulose in Betracht gezogen werden, bei denen ein angem. Bhdlgs.verlauf nicht bestätigt werden kann. Pat. sollen angewiesen werden, mediz. Rat einzuholen, wenn Anzeichen od. Sympt. e. Infektion auftreten. Bei anaphylakt. od. and. schweren Überempfindl.reakt. soll d. Verabreichg. v. STELARA* sofort abgebrochen u. e. geeign. Therapie eingeleitet werden. B. Auftr. e. exfoliat. Dermatitis (Erythrodermie) od. erythroderm. Psoriasis muss e. angemess. Ther. eingeleitet u. be V. a. eine Arzneimittelreakt. STELARA" abgesetzt werden Lebendvirus- od. Lebendbakterienimpfstoffe (z. B. Bazillus Calmette-Guérin (BCC)) sollen nicht gleichzeitig m. STELARA verabreicht werden. Vor e. Impfung muss $d$. Anw. von STELARA* mind. 15 Wo. ausgesetzt werden u. kann frühestens 2 Wo. danach wieder aufgenommen werden. Vorsicht, wenn gleichzeitig and. Immunsuppressiva angewendet werden od. wenn e. Wechsel v. and. biolog. Immunosuppressiva erwogen wird. Ältere Pat.. Immuntherapie gg. e. Allergie. Pat. m. Latexüberempfindl.. Nebenwirk.: Erw. Pat.: Häufig: Infekt. d. oberen Atemw., Nasopharyng. Schwindel, Kopfschm., Oropharyngeale Schmerzen, Diarrhö, Nausea, Erbrechen, Pruritus, Rückenschm., Myalgie, Arthralgie, Müdigk., Erythem an d. Injekt.stelle, Schmerzen an d. Injekt.stelle. Gelegentl.: Cellulitis, dentale Infekt., Herpes zoster, Virusinfekt. d. oberen Atemw., vulvovag. Pilzinfektionen, Überempfindl. reakt. (einschl. Hautausschlag, Urtikaria), Depress., Facialisparese, verstopfte Nase, Exfoliat. $d$. Haut, Akne, pustulöse Psoriasis, Reakt. an d. Injekt.stelle (einschl. Hämorrhagie, Hämatom, Verhärtung, Schwellung u. Pruritus), Asthenie. Selten: schwerwiegende Überempfindl.reakt. (einschl. Anaphylaxie, Angioödem), exfoliat. Dermatitis (Erythrodermie). Pädiatr. Pat.: Die in e. Phase III-Studie berichteten unerwünscht. Ereign. waren denjen. ähnlich, d. in vorangeg. Studien b. Erw. m. Plaque-Psoriasis beob, wurden. Warnhinw.: Alle Pat, bes, diejenigen üb, 60 Jahre sow. Pat. $m$. einer läng, immunsuppr. Therapie od. PUVA-Bhdlg, in der Anamnese, sollten hinsichtl. d. Auftretens v. nicht-melanozytärem Hautkrebs überwacht werden. Währ. d. Anw. u. für mind. 15 Wo. danach zuverlässige Verhütungsmethoden anwenden. Arzneimittel für Kinder unzugängl. aufbew.. Stand d. Inform.: 10/2016. Verschreibungspflichtig. Pharmaz. Unternehmer: Janssen-Cilag International NV, Turnhoutseweg 30, B 2340 Beerse, Belgien. Örtl. Vertreter: Janssen-Cilag CmbH, Johnson \& Johnson Platz 1, 41470 Neuss. 


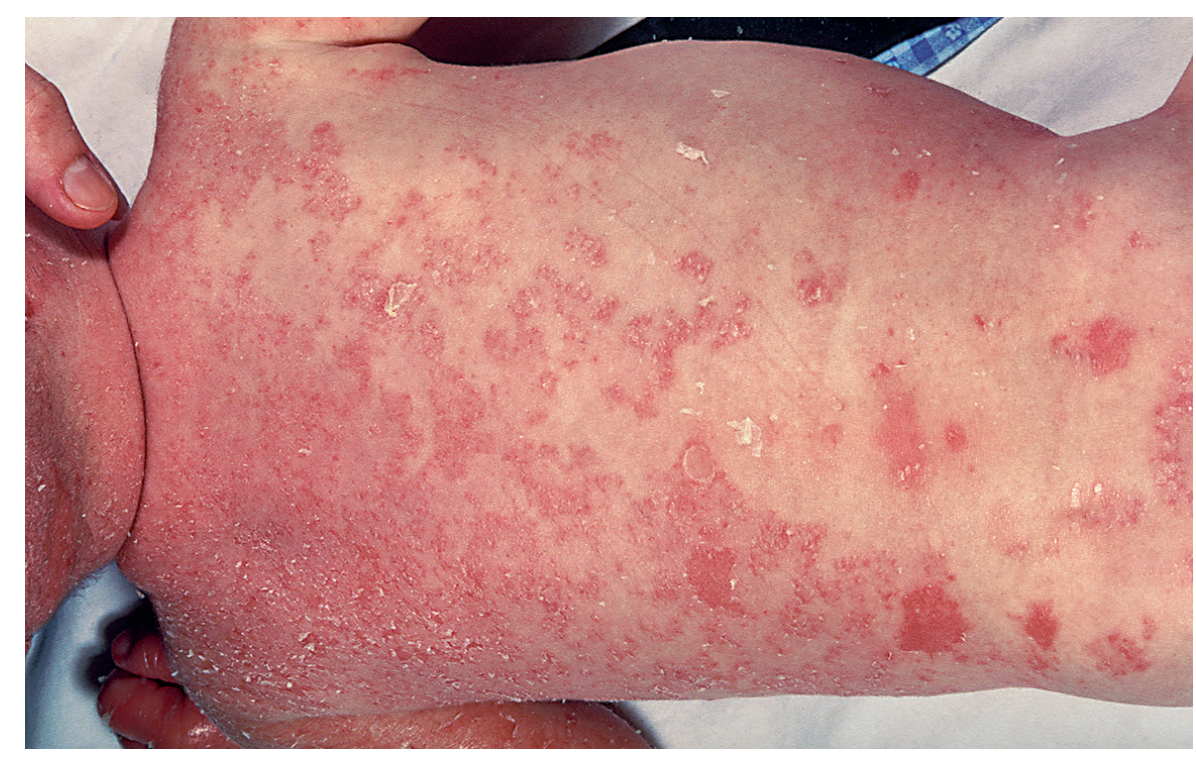

Eine weitere ekzematöse Erkrankung ist die infantile seborrhoische Dermatitis, die wie die atopische Dermatitis im frühen Kindesalter vorkommt. Der Juckreiz ist nur leicht oder fehlt ganz, und anders als die atopische Dermatitis tritt die Krankheit bevorzugt im Windelbereich auf. Dieser Bereich bleibt bei der atopischen Dermatitis aufgrund von Hyperhydratation durch die Windel typischerweise frei von Hautveränderungen. Es gibt eine Vielzahl anderer Differenzialdiagnosen [15], darunter weitere entzündliche Hauterkrankungen wie Psoriasis und Immunschwächeerkrankungen wie das Omenn-Syndrom und das Wiskott-AldrichSyndrom, maligne Erkrankungen wie Langerhans-Zell-Histiozytose und parasitäre Erkrankungen wie Skabies. Alle diese Differenzialdiagnosen erfordern unterschiedliche therapeutische Maßnahmen.

Bei Patienten mit atopischer Dermatitis ist die wichtigste therapeutische Maßnahme die regelmäßige Anwendung von Emollientien, um die defekte Hautbarriere wiederherzustellen [16]. Dies kann eine erneute Verschlimmerung der Erkrankung verhindern und dabei helfen, den Einsatz von Kortikosteroiden zu vermeiden $[17,18]$. Es hilft auch, den Juckreiz zu verringern [19].

Neben der Anwendung von Feuchtigkeitscremes sind topische entzündungshemmende Arzneimittel (topische Kortikosteroide und Calcineurin-Inhibitoren) Bestandteile der Standardtherapie bei Patienten mit atopischer Dermatitis. Sie dienen sowohl der reaktiven als auch der proaktiven Therapie zur Kontrolle der Hautentzündungen. Dies wurde auch für Kinder gut dokumentiert [20]. Obwohl topische Kortikosteroide immer noch die Therapie der Wahl darstellen [21], sind Calcineurin-Inhibitoren für die Behandlung der atopischen Dermatitis bei Kindern angezeigt. Sie werden für den Einsatz in empfindlichen Bereichen wie Gesicht, Hautfalten und den Genitoanalbereich empfohlen und verfügen über antipruritische Eigenschaften [22]. Darüber hinaus sind sie in Fällen von Intoleranz gegenüber Kortikosteroiden angezeigt. Kürzlich wurde noch einmal hervorgehoben, dass CalcineurinInhibitoren sicher sind, auch bei der Behandlung von Kindern. Auch wenn sie in vielen Ländern, darunter auch Deutschland, nicht für eine Behandlung vor dem 2. Lebensjahr zugelassen sind, gibt es viele Studien, die ihre Wirksamkeit und Sicherheit im Kleinkindalter gezeigt haben [23, 24].

Wenn Patienten trotz Berücksichtigung der auslösenden Faktoren nicht auf die topische Therapie reagieren, ist eine systemische Therapie angezeigt. Ciclosporin (zugelassen ab 16 Jahren) ist das einzige Medikament, das in Deutschland zugelassen ist; Mycophenolat-Mofetil, Methotrexat und Azathioprin sind Medikamente, die auch effektiv, aber für die Indikation atopische Dermatitis nicht zugelassen sind (Off-Label-Use). Alle besitzen antipruritische Eigenschaften.

Kürzlich hat eine kanadische Gruppe [25] auf die hervorragende antipruritische Wirkung der Kombination aus Clonidin (adrenerger Agonist) und Trimeprazin (Phenothiazin) bei einem 6-jährigen Jungen mit schwerer atopischer Dermatitis und refraktärem Pruritus hingewiesen. Auch andere interessante antipruritische Arzneimittel befinden sich in der Entwicklung. Draelos et al. [11] zeigten die positive Wirkung des topischen Phosphodiesterase-4-Inhibitors Crisaborol bei Kindern und Jugendlichen mit leichter bis mittelschwerer atopischer Dermatitis basierend auf Daten aus 4 Studien. Der Schweregrad des Pruritus wurde mithilfe einer 4-Punkte-Skala beurteilt. Die Therapie führte zu einer statistisch signifikanten Reduktion des Pruritus-Schweregrades bei den Messungen an den Tagen 8 und 29. Darüber hinaus befinden sich viele neue Medikamente, insbesondere Biologika, in der klinischen Studienphase [26]. Zusätzlich zum klinischen Schweregrad wird auch die Schwere des Pruritus bewertet. Auf die Studien an Erwachsenen folgen Studien an Kindern; beispielsweise laufen bereits Studien an Kindern mit Dupilumab, einem gegen die $\alpha$-Untereinheit der Interleukin (IL)-4- und IL-13-Rezeptoren gerichteten monoklonalen Antikörper [27].

Es gibt viele bekannte auslösende Faktoren für die atopische Dermatitis, die mit Pruritus assoziiert ist. Kontaktallergene sind einer dieser Faktoren. De Waard-van der Spek und Oranje [28] zeigten, dass 55\% der Kinder mit positiven Reaktionen auf ein oder mehrere Kontaktallergene an atopischer Dermatitis litten. Sie empfeh- 
Tab. 2. Juckende exanthematöse Krankheiten (Auswahl)

\begin{tabular}{|c|c|c|}
\hline Krankheiten & Klinisches Muster & Alter bei Krankheitsbeginn \\
\hline $\begin{array}{l}\text { Exantheme im frühen Kindesalter } \\
\text { Infantile eosinophile pustulöse } \\
\text { Follikulitis }\end{array}$ & $\begin{array}{l}\text { chronischer rezidivierender Verlauf der } \\
\text { vesikulopustulären Läsionen auf der Kopfhaut }\end{array}$ & Kleinkindalter \\
\hline Infantile Akropustulose & $\begin{array}{l}\text { chronischer rezidivierender Verlauf der } \\
\text { vesikulopustulären Läsionen an Händen und Füßen }\end{array}$ & Kleinkindalter \\
\hline \multicolumn{3}{|l|}{$\begin{array}{l}\text { Exanthem aufgrund eines direkten } \\
\text { Viruseffekts }\end{array}$} \\
\hline Varizellen & $\begin{array}{l}\text { polymorphe Exantheme, sekundäre Impetigo- } \\
\text { Entwicklung, Beteiligung von Kopfhaut und } \\
\text { Mundschleimhaut }\end{array}$ & vor allem im Kleinkindalter \\
\hline \multicolumn{3}{|l|}{ Parainfektiöse Virusexantheme } \\
\hline $\begin{array}{l}\text { Papulopurpurisches Handschuh- } \\
\text { Socken-Syndrom }\end{array}$ & $\begin{array}{l}\text { Ödem und Erythem mit Petechien an Händen } \\
\text { und Füßen, Enanthem }\end{array}$ & $\begin{array}{l}\text { Jugendliche und junge } \\
\text { Erwachsene }\end{array}$ \\
\hline Pityriasis rosea & $\begin{array}{l}\text { Primärmedaillon, Exanthem entlang der Langer- } \\
\text { Linien, Collarette-Schuppung aller Läsionen }\end{array}$ & Jugendliche \\
\hline Gianotti-Crosti-Syndrom & $\begin{array}{l}\text { monomorphe Papeln oder Papulovesikel auf } \\
\text { Wangen, Extremitäten und Gesäß }\end{array}$ & Kleinkinder und Vorschüler \\
\hline
\end{tabular}

len, auch an die Möglichkeit der Kontaktallergie zu denken und den Epikutantest zu veranlassen. Auch andere Autoren weisen auf die diagnostische Bedeutung von Epikutantests bei refraktären Formen der atopischen Dermatitis hin [29].

\section{Kontaktdermatitis}

Eine allergische Kontaktdermatitis kommt bei Kindern häufiger vor als bisher angenommen, auch bei Kindern sehr geringen $\mathrm{Al}-$ ters [30]. Im Zeitraum von 2002 bis 2008 wurden bei 321 Kindern unter 3 Jahren mit Verdacht auf allergische Kontaktdermatitis Epikutantests durchgeführt. Die häufigsten positiven Reaktionen gab es bei Metallen, Cocamidopropylbetain, Neomycin und Methylchlorisothiazolinon/Methylisothiazolinon. Dabei bestand kein Unterschied in der Prävalenz der Kontaktsensibilisierung zwischen Kindern mit und ohne atopische Dermatitis.

Vor allem im Kindesalter sind die folgenden Quellen für mögliche allergische oder irritative toxische Kontaktdermatitis $\mathrm{zu}$ berücksichtigen: temporäre Henna-Tattoos und Haarfärbemittel (häufige Auslöser: p-Phenylendiamin, p-Toluylendiamin), Spielekonsolen (häufigste Auslöser: Kautschukbestandteile), Schienbeinschützer und Bandagen (häufigste Auslöser: Kautschukbestandteile, Schweiß, Reibung), Mobiltelefone und Tablets (häufigster Auslöser: Nickel) und Kosmetika (häufige Auslöser: Duftstoffe, Konservierungsmittel wie Methylisothiazolinon, Naturkosmetik) [31].

\section{Exantheme}

Insbesondere im frühen Kindesalter kommen extrem juckende Exantheme unbekannter Ursache vor. Dazu gehören die infantile eosinophile pustulöse Follikulitis und die infantile Akropustulose (Tab. 2).

\section{Infantile eosinophile pustulöse Follikulitis}

Die infantile eosinophile pustulöse Follikulitis ist ein typischer, aber seltener vorkommender Hautausschlag im frühen Kindesalter [32]. Er tritt vornehmlich auf der Kopfhaut auf mit der Entwicklung von extrem juckenden vesikulopustulären Läsionen. Ein charakteristischer Befund ist ein chronisch-rezidivierender Verlauf, der bis zu 3 Jahre andauern kann. In diesem Alter sind Differenzialdiagnosen zu berücksichtigen, die ebenfalls mit juckenden vesikulopustulären Hautausschlägen einhergehen. Von diesen ist die infantile Skabies häufig, weit verbreitet und befällt im Gegensatz zu älteren Kindern und Erwachsenen auch die Kopfhaut. Andere Differenzialdiagnosen sind sehr selten und umfassen die infantile Akropustulose (siehe unten), Incontinentia pigmenti (entlang der Blaschko-Linien angeordnet, in der Regel nicht juckend) und die Langerhans-Zell-Histiozytose, für die eine Biopsie zur histologischen/immunhistochemischen Analyse erforderlich ist [33]. Umstritten bleibt, ob die infantile eosinophile pustulöse Follikulitis eine Variante der klassischen eosinophilen pustulösen Follikulitis nach Ofuji [34] bei Erwachsenen ist. Sonderformen der eosinophilen pustulösen Follikulitis sind auf AIDS (erworbenes Immunschwäche-Syndrom), Medikamente, Silikoninjektionen und Leukämie zurückzuführen [35]. Sie werden häufiger bei Erwachsenen als bei Kindern beobachtet [35].

Der unerträgliche Juckreiz ist äußerst belastend. Die Therapie der Wahl ist eine Kombination aus topischen Kortikosteroiden und Antihistaminika [36]. Als Alternative können topische Calcineurin-Inhibitoren verwendet werden $[37,38]$.

\section{Infantile Akropustulose}

Die infantile Akropustulose tritt vor allem bei älteren Säuglingen und Kleinkindern auf [39] und häufiger bei schwarz- als bei weißhäutigen Patienten [40]. Wie die infantile eosinophile pustulöse 
Abb. 3. Infantile Akropustulose mit zahlreichen Vesikulopusteln.

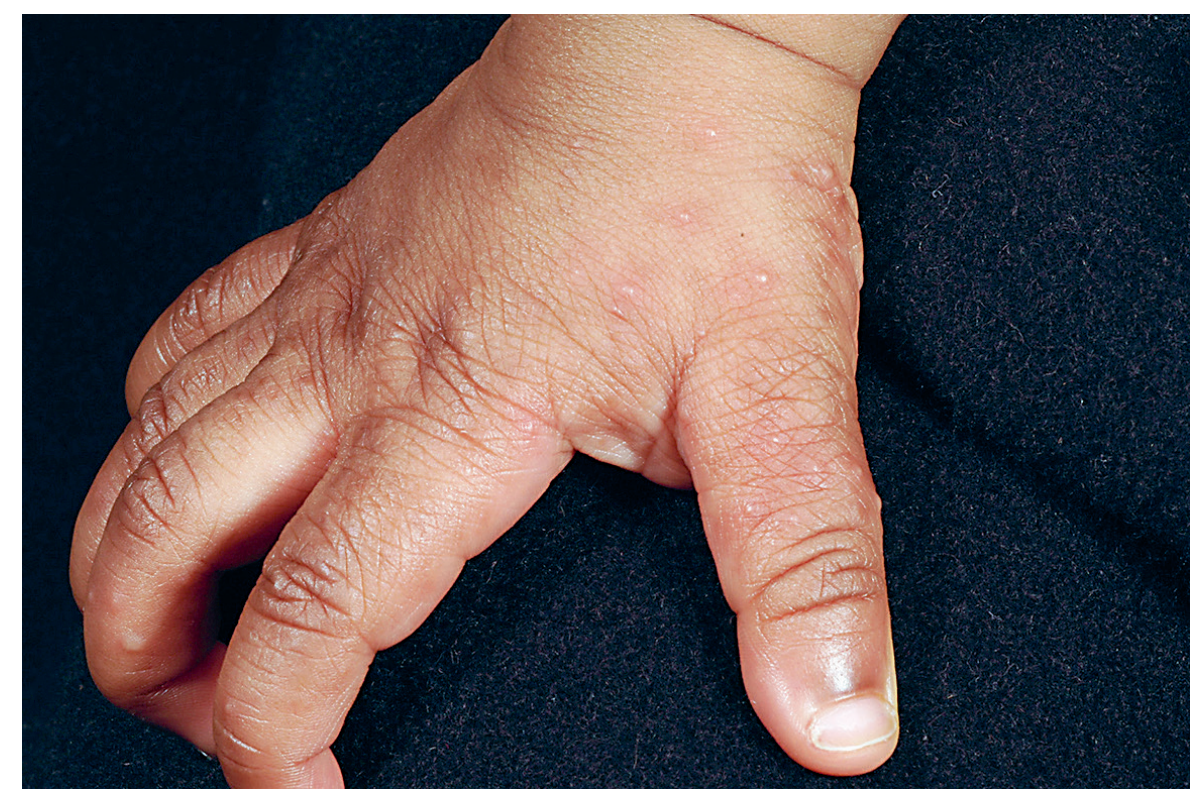

Follikulitis kommt sie selten vor und zeigt einen rezidivierenden Verlauf mit einem stark juckenden vesikulopustulösen Hautausschlag. Sie ist jedoch nicht follikulär gebunden und betrifft typischerweise die Hände und Füße (Abb. 3). Einige Autoren sind der Ansicht, dass der Ausschlag auf eine persistierende Überempfindlichkeit des Immunsystems von Kleinkindern, die erfolgreich wegen eines Skabies-Befalls behandelt wurden, zurückzuführen ist [40-43]. Über diese Hypothese besteht allerdings kein Konsens. Die sterilen subkornealen Pusteln enthalten neutrophile und eosinophile Granulozyten. Differenzialdiagnosen umfassen Skabies, Infektionen wie Impetigo und Candidiasis sowie die transiente neonatale pustulöse Melanose [44]. Darüber hinaus sollte die lokale Form der Defizienz des IL-1-Rezeptor-Antagonisten (DIRA) in Betracht gezogen werden, wenn sterile Pusteln an Händen und Füßen vorhanden sind [45].

Aufgrund des extremen Juckreizes sind Kinder mit infantiler Akropustulose sehr unruhig und benötigen eine angemessene Therapie. Topische Kortikosteroide wirken entzündungshemmend und auch antipruritisch. Kommt es nicht zu einem Ansprechen, können sie mit Antihistaminika kombiniert werden. In einigen seltenen Fällen ist eine systemische Behandlung erforderlich. Die Therapie der Wahl ist Dapson, das den Juckreiz reduziert, jedoch solltenNebenwirkungen wie hämolytische Anämie und Methämoglobinämie beachtet werden.

Generell kommen Exantheme im Kindesalter sehr häufig vor und treten hauptsächlich im Rahmen von Virusinfektionen auf. Viele Viren zeigen einen direkten Effekt auf die Haut (z.B. Varizellen), während andere Exantheme als Antwort des Immunsystems auf eine Virusinfektion aufzufassen sind (z.B. das Gianotti-CrostiSyndrom). Letztere werden auch als parainfektiöse Virusexantheme bezeichnet [46].

\section{Varizellenexanthem (Exanthem aufgrund der direkten} Wirkung des Varizella-Zoster-Virus auf Haut und Schleimhäute) Varizellen (Windpocken) sind die initiale Manifestation des
Varizella-Zoster-Virus (HHV-3). Die Infektion tritt häufig im Kindesalter auf, jedoch gibt es eine Verschiebung hin zu Jugendlichen und jungen Erwachsenen. Das juckende polymorphe Exanthem (Ausbruch neuer Effloreszenzen, von Maculae zu Papeln, Papulovesikeln, Bläschen, Pusteln und Krusten) breitet sich vom Haaransatz nach kaudal zu den unteren Extremitäten aus. Die Beteiligung der Kopfhaut und Mundschleimhaut ist ein charakteristischer Befund. Die Krankheit geht mit erhöhter Temperatur und leichter Müdigkeit einher.

Kratzen fördert Sekundärinfektionen mit Streptococcus pyogenes und Staphylococcus aureus. Zu den Komplikationen zählen lokale und systemische Infektionen und eine neurologische Beeinträchtigung.

Therapeutisch ist es sehr wichtig, Sekundärinfektionen als Komplikation zu verhindern. Insbesondere bedeutet dies, den Juckreiz symptomatisch mithilfe von z.B. Gerbstoffen oder Lotio alba/Calamin-Lotion, auch kombiniert mit Antihistaminika zu lindern. In schweren Fällen, insbesondere bei immungeschwächten Kindern, können antivirale Medikamente wie Acyclovir oder alternativ Famciclovir und Valacyclovir eingesetzt werden [47].

Handschuh-Socken-Syndrom (parainfektiöses Virusexanthem) Das Handschuh-Socken-Syndrom ist am häufigsten Parvovirus B-assoziiert, seltener mit anderen Viren wie Cytomegalievirus, Coxsackie-B6-Virus und Masernviren [48]. Das Exanthem tritt vor allem bei Jugendlichen und jungen Erwachsenen auf und ist durch Ödeme und Erytheme der Hände und Füße gekennzeichnet, die sehr purpuriform und petechial sein können. Die Läsionen sind von intensivem Juckreiz und Brennen begleitet. Darüber hinaus weisen viele Patienten ein orales Enanthem mit Petechien und Erosionen auf. Häufig sind auch Fieber und Arthralgie damit verbunden. Die Krankheit ist selbstlimitierend und erfordert lediglich eine symptomatische Behandlung. 

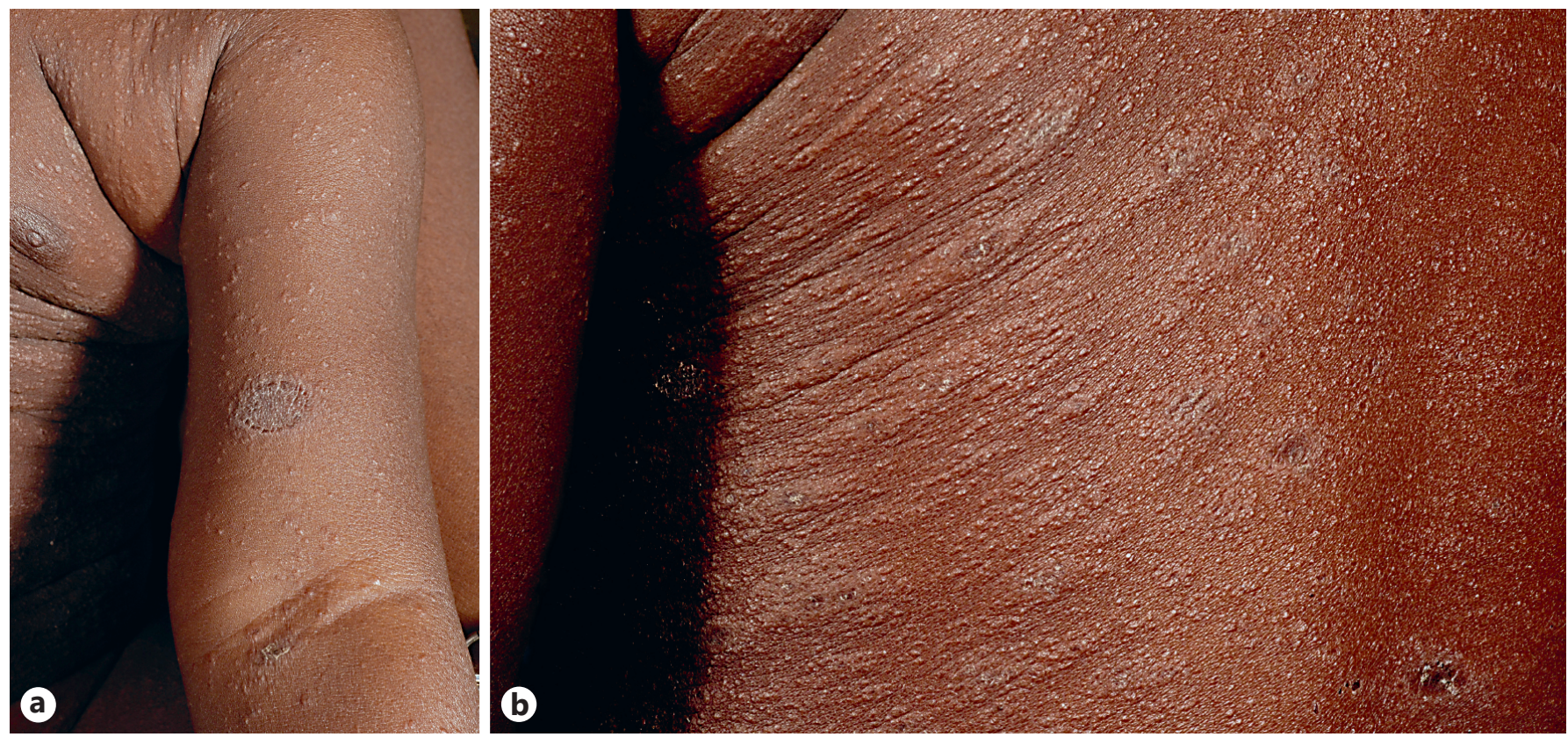

Abb. 4. Pityriasis rosea: (a) Primärmedaillon und (b) Exanthem entlang der Langer-Linien.

Pityriasis rosea (parainfektiöses Virusexanthem)

Pityriasis rosea ist ein häufig auftretendes Exanthem und betrifft vor allem Jugendliche und junge Erwachsene. Die Erkrankung ist hauptsächlich mit den humanen Herpesviren 6 und 7 assoziiert [49]. Das klinische Bild zeigt ein typisches Muster: ein Primärmedaillon (Herald-Patch), dem ein Exanthem folgt, das entlang der Hautspaltlinien (Langer-Linien) verläuft. Primäre und sekundäre Läsionen präsentieren sich als ovuläre rotbraune Plaques mit typischer Collerette-Schuppung (Abb. 4). Das Exanthem befindet sich überwiegend am Rumpf und an den proximalen Extremitäten. Die Patienten zeigen eine extrem reizbare Haut, die sich besonders nach dem Baden bemerkbar macht und zu Ekzemen und starkem Pruritus führen kann [50]. Daher sollten Hautreizungen durch zu kräftiges Waschen und den übermäßigen Gebrauch von Seife vermieden werden. Wenn sich die Patienten an die Empfehlungen halten, benötigen viele von ihnen keine spezifische Therapie. In schweren Fällen gibt es verschiedene Therapieoptionen: Kombination von milden topischen Glukokortikoiden mit niedrig dosierter UVB-Strahlung (für Patienten ab 12 Jahren) [50], Erythromycin [51] und Acyclovir [52].

\section{Gianotti-Crosti-Syndrom (parainfektiöses Virusexanthem)}

Das Gianotti-Crosti-Syndrom ist ein insbesondere bei Kleinkindern auftretendes Exanthem, das hauptsächlich mit dem EpsteinBarr-Virus assoziiert ist. An den bevorzugt betroffenen Stellen, den Wangen, Extremitäten und dem Gesäß (Abb. 5), weisen die Kinder ein monomorphes Exanthem auf, das aus kleinen Papeln oder Papulovesikeln besteht. Das Exanthem kann von Pruritus begleitet sein. Im Allgemeinen sollten Emollientien angewendet werden. In Fällen mit schwerem Pruritus kann ein Antihistaminikum in Betracht gezogen werden [53]. In refraktären Fällen kann Ribavirin eine Option darstellen [54].
Infestationen

Skabies

Skabies ist eine in allen Altersgruppen weit verbreitete Infestation. Nach einer Sensibilisierungsphase von 2-3 Wochen sind beim primären Befall an den bevorzugten Stellen der Beugeseite der Handgelenke, der Achselfalten und Interdigitalzonen der Hände Hautläsionen zu beobachten. Sie bestehen aus ekzematisierten Läsionen mit Knötchen, Bläschen und Schuppungen und werden durch eine zelluläre Immunantwort auf Sarcoptes scabiei-Milben sowie deren Eier und Ausscheidungen verursacht. Die Immunantwort ist auch die Ursache des Pruritus, der nachts am stärksten ist [55]. Krankheitstypisch sind kutane kommaartige Gänge, die von den Milbenweibchen in die Hornschicht gegraben werden. Anders als bei älteren Kindern und Erwachsenen sind bei Patienten im frühen Kindesalter auch Gesicht und Kopf betroffen. Darüber hinaus neigen Säuglinge und Kleinkinder eher dazu, polymorphe ekzematöse Läsionen mit Vesikel, Papeln, Pusteln und Schuppung zu entwickeln [56]. Zusätzlich weisen sie typischerweise Pusteln in der Fußsohlenregion auf. Die klinische Skabies-Diagnose kann durch die mikroskopische und/oder dermatoskopische Untersuchung auf Milben, Eier und/oder Gänge bestätigt werden. Die wichtigste Differenzialdiagnose ist die atopische Dermatitis (auch polymorphe ekzematöse Läsionen), es kann jedoch auch eine infantile Akropustulose, eine infantile eosinophile Follikelpustulose oder eine Langerhans-Zell-Histiozytose vorliegen. 5\%iges Permethrin ist die Standardtherapie. Nach einer erfolgreichen Behandlung weisen einige Patienten stark juckende rötlich-braune Papeln/Knötchen auf, die frei von Skabies-Milben sind. Dabei handelt es sich um postskabiöse Granulome, die auf topische Kortikosteroide der Klasse 2 gut ansprechen, und in manchen Fällen genügen auch Emollientien. 


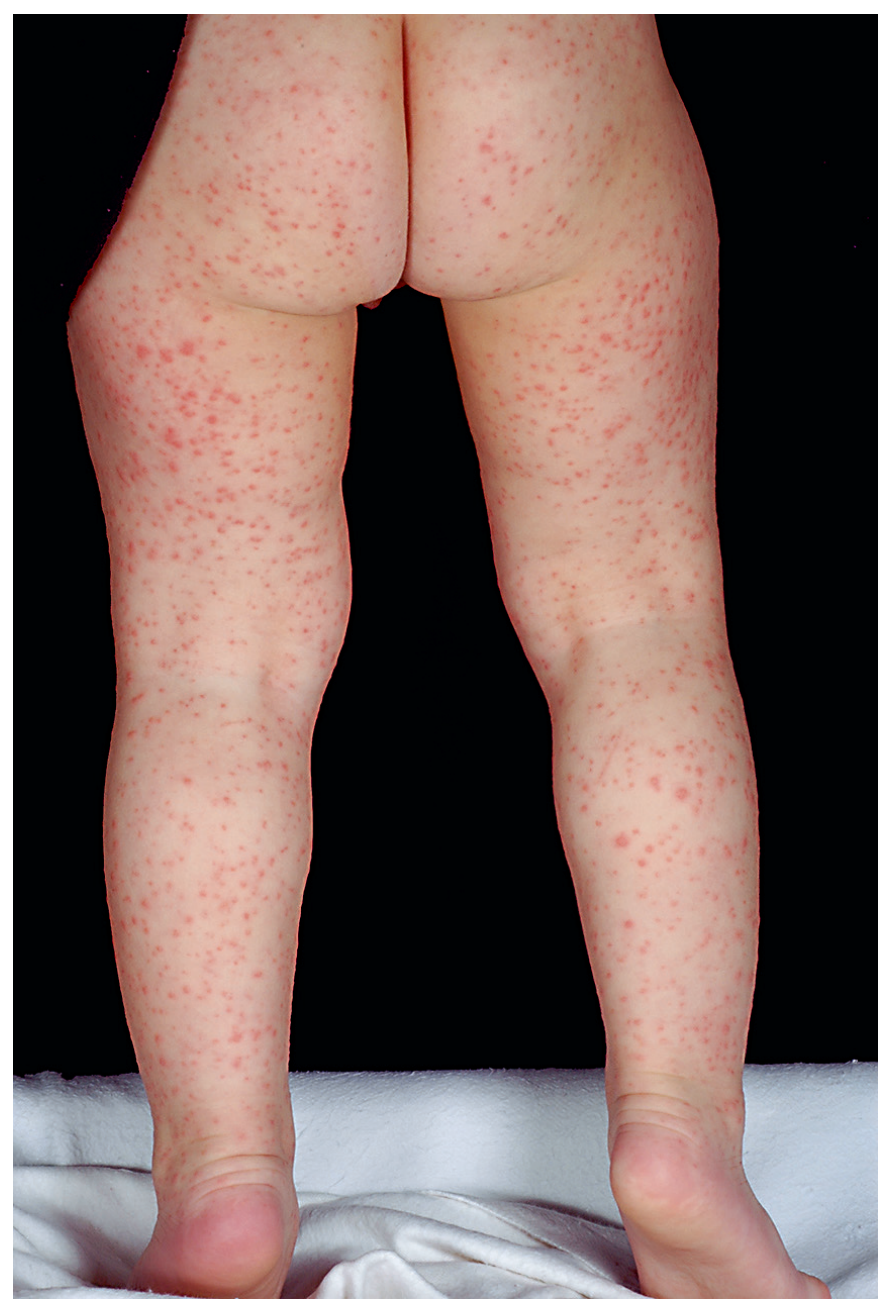

Abb. 5. Gianotti-Crosti-Syndrom, monomorpher Aspekt der Papulovesikel.

\section{Pediculosis capitis}

Pediculosis capitis, die durch einen Befall mit Pediculus humanus capitis (Kopfläuse) verursacht wird, ist die häufigste Form eines Läusebefalls und betrifft hauptsächlich Schulkinder. Die Übertragung erfolgt von Kopf zu Kopf. Eine Übertragung durch Gegenstände wie Kämme, Bürsten oder Handtücher ist selten. Pruritus ist das häufigste klinische Symptom und ist auf eine zelluläre Immunreaktion auf Proteine im Speichel der Läuse zurückzuführen. Patienten reagieren mit Kratzen, wodurch sich das Risiko einer bakteriellen Infektion mit Staphylococcus aureus oder Streptococcus pyogenes erhöht. Dies kann zur Entwicklung einer Impetiginisierung und zu einer zervikalen und subokzipitalen Lymphadenopathie führen [57]. Die bevorzugte Therapie sind topische Pyrethroide wie Permethrin oder Silikonöl. Kürzlich wurden Studien veröffentlicht, die eine Resistenz von Kopfläusen gegenüber Pyrethroiden in Europa zeigten [58]. Jedoch korrelierte die molekulare Resistenz (Mutation im Gen für die a-Untereinheit des spannungsabhängigen Natriumkanals, das sogenannte knockdown resistance ( $k d r)$-Gen) nicht mit dem Versagen von Permethrin oder Pyrethrin bei der Behandlung von Pediculosis capitis bei Kindern in Deutschland [58]. Die Möglichkeit einer Resistenz sollte jedoch bedacht werden.

\section{Zerkariendermatitis}

Die Zerkariendermatitis (Badedermatitis) ist eine Überempfindlichkeitsreaktion der Haut auf nichthumane SchistosomenParasiten, die in die Haut eindringen. Dies geschieht beim Schwimmen oder Waten in Seen oder Teichen und wird durch die Freisetzung proteolytischer Enzyme erleichtert [59]. Die wichtigsten Parasiten sind die Spezies der Gattung Trichobilharzia [60]. Die eigentlichen Wirte sind Wasservögel, die die Eier der Parasiten mit dem Kot ins Wasser ausscheiden. Aus den Eiern schlüpfen die Larven (Mirazidien), die in Wasserschnecken (Zwischenwirte) eindringen. Nach der Entwicklung zu den Zerkarien verlassen sie die Schnecke und suchen erneut nach ihren eigentlichen Wirten. Allerdings befallen sie irrtümlich auch Menschen, die Endwirte sind. Die Zerkarien sterben im menschlichen Stratum corneum, da sie nicht in der Lage sind, die Blutgefäße zu erreichen.

An den betroffenen Hautstellen entwickeln die Patienten stark juckende Papeln und Papulovesikel, die einige Stunden nach dem Kontakt mit den Zerkarien auftreten (Abb. 6). Zur Linderung des Juckreizes sind Antihistaminika und topische Kortikosteroide hilfreich.

Es sind auch einige andere juckende Dermatosen in Betracht zu ziehen: Insektenstiche, humane Bilharziose (vor allem in Afrika, Asien und Südamerika), Kontaktdermatitis (insbesondere durch den Kletternden Giftsumach (Giftefeu)) und QuallenDermatitis.

\section{Juckreiz im Windelbereich im Kindesalter}

Viele Dermatosen im Windelbereich sind von Juckreiz begleitet. Dazu gehören entzündliche Dermatosen (z.B. Psoriasis), Infektionen/Parasitosen (z.B. bullöse Impetigo, Candidiasis), Stoffwechselstörungen (z.B. Acrodermatitis enteropathica), Autoimmunerkrankungen (z.B. Lichen sclerosus) und Neoplasien (z.B. Langerhans-Zell-Histiozytose). Diese sind in Tabelle 3 aufgeführt. Der folgende Abschnitt konzentriert sich auf den Lichen sclerosus.

\section{Genitaler Lichen sclerosus}

Der genitale Lichen sclerosus ist eine seltene Autoimmunerkrankung, von der vor allem erwachsene Frauen betroffen sind. Mädchen vor der Menarche sind in geringerem Ausmaß betroffen. Auch bei Jungen ist Lichen sclerosus bekannt und mit einer erworbenen Vorhautverengung (Phimose) assoziiert [61]. Das Hauptmerkmal der Dermatose ist der unerträgliche Juckreiz, der erhebliche Auswirkungen auf die Lebensqualität hat [62]. Die Dermatose zeigt ein typisches 8-förmiges Muster im Bereich um die Vulva und den Anus [63]. Porzellanweiße Papeln und Plaques sind morphologisch ausgeprägt. Die Haut des Lichen sclerosus ist äußerst fragil, und durch Reiben oder Kratzen werden Blutungen und Fissuren verursacht [62]. Die hämorrhagischen Hautläsionen werden nicht selten als sexueller Missbrauch fehlgedeutet. Weitere Differenzialdiagnosen beinhalten Infektionen und Ekzeme (Kontaktekzem, atopische Dermatitis) [63] (Tab. 1). 


\section{Ekzem-Therapie: Schnelle Symptomlinderung zählt}

\section{Optimale Nutzen-Risiko-Relation}

Therapeutischer Index 2

Höchste galenische Vielfalt

5 Galeniken

Schnelle Juckreizlinderung

PRURITUS-NIMPA-Studie'

$\operatorname{roc}(6$

(c)
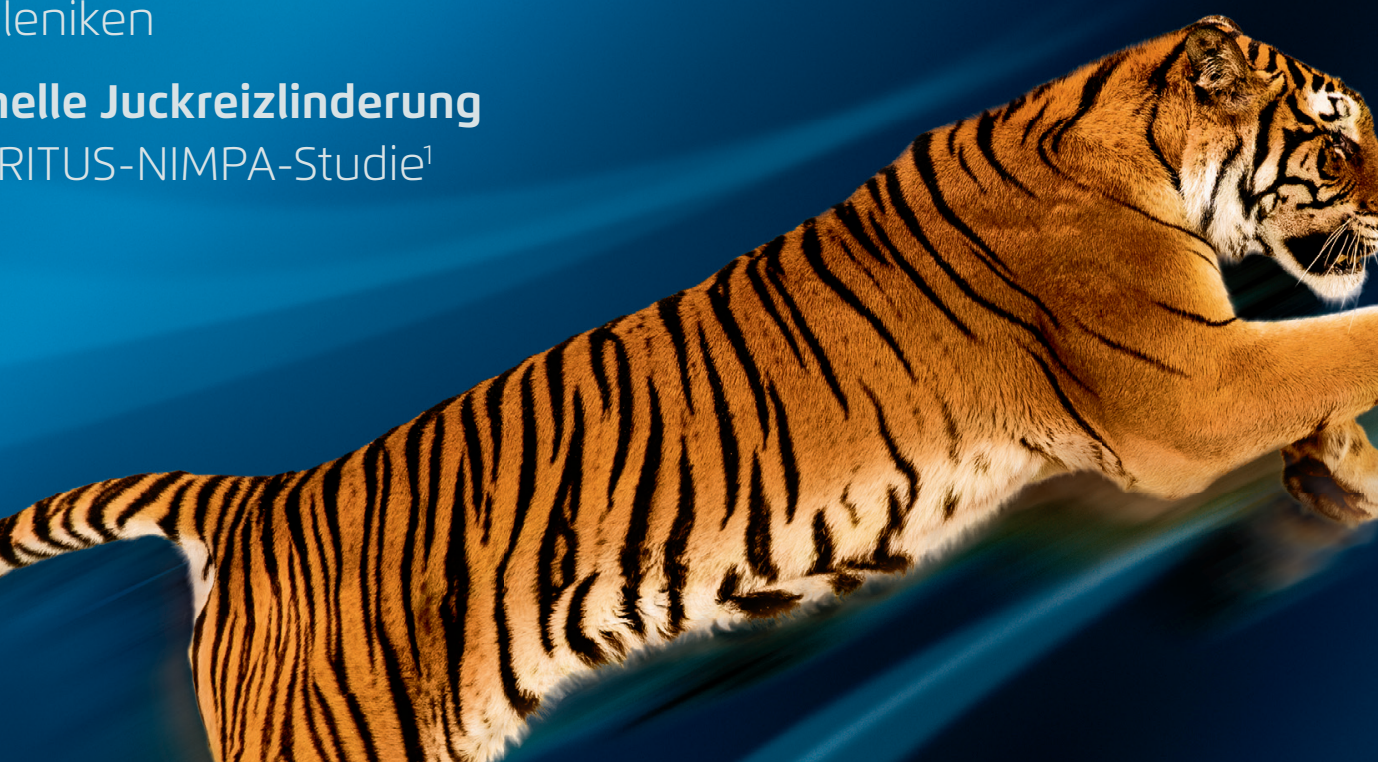

\section{a Advantan Stark ohne Kompromisse}

www.jenapharm.de

* Curto et al., J Eur Acad Dermatol Venereol. $2014 ; 2(10): 1356$ - 62.

Advantan' Milch 0,1\%, Advantan $0,1 \%$ Salbe, Advantan $0,1 \%$ Fettsalbe, Advantan $0,1 \%$ Creme, Advantan $0,1 \%$ Lösung

Wirkstoff: Methylprednisolonaceponat. Vor Verschreibung Fachinformation beachten. Zusammensetzung: Arzneilich wirksame Bestandteile: $1 \mathrm{~g}$ Advantan Milch, Salbe, fettsalbe, Creme, bzW. $1 \mathrm{ml}$ Advantan Lösung enthält jeweils $1 \mathrm{mg}(0,1 \%)$ Methylprednisolonaceponat. Sonstige Bestandteile: Milch: mittelkettige Triglyceride, Glyceroltrialkanoat $\left(\mathrm{C}_{8}-\mathrm{C}_{18}\right)$, Macrogol-2-stearylether, Macrogol-21stearylether, Benzylalkohol, Natriumedetat, Glycerol $85 \%$, ger. Wasser. Salbe: Dioctadecylcitrat-Pentaerythritoldicocoat[3-hydroxy-3,4-bis(octadecyloxycarbonyl)butanoat]-BienenwachsAluminiumstearat-Gemisch, dickflüss. Paraffin, gebleicht. Wachs, ger. Wasser, weißes Vaselin. Fettsalbe: dickflüss. Paraffin, hydriert. Rizinusöl, mikrokrist. Kohlenwasserstoffe $\left(C_{40}-C_{50}\right)$, weißes Vaselin. Creme: Benzylalkohol, Butylhydroxytoluol, Cetylstearylalkohol, Decyloleat, ger. Wasser, Glycerol $85 \%$, Glycerolmonostearat 40-55, Glyceroltrialkanoat $\left(\mathcal{C}_{8}-C_{18}\right)$, Hartfett, Macrogolstearat 2000 Natriumedetat. Lösung: Isopropylmyristat, 2-Propanol. Anwendungsgebiete: Milch: leichte bis mittelschwere akute exogene Ekzeme (allerg. Kontaktdermatitis, irritative Kontaktdermatitis, Natriumedetat. Lösung: Isopropylmyristat, 2-Propanol. Anwendungsgebiete: Milch: leichte bis mittelschwere akute exogene Ekzeme (allerg. Kontaktdermatitis, irritative Kontaktdermatitis,
nummuläres Ekzem, dyshidrotisches Ekzem, Eczema vulgaris), endogenes Ekzem (atopische Dermatitis, Neurodermitis), stark entzündetes seborrhoisches Ekzem. Salbe, Fettsalbe, Creme: endogenes Ekzem (atopische Dermatitis, Neurodermitis), Kontaktekzem, degeneratives Ekzem und nummuläres Ekzem. Lösung: entz. und prurit. Dermatosen der Kopfhaut, z.B. atopische Dermatitis

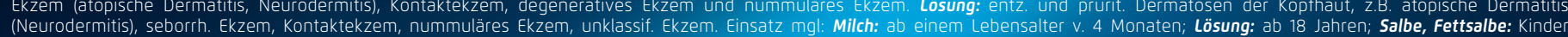
Jugendliche und Erwachsene; Creme: ab einem Lebensalter v. 3 Jahren; Gegenanzeigen: Uberempfindlichkeit gegen den Wirkstoff o. einen der sonst. Bestandteile, tuberkul. u. syphilitische Prozesse virale Infektionen, Rosazea, periorale Dermatitis, Ulzera, Acne vulgaris, atroph. Hautkrankh., Impfreaktionen im Behandlungsbereich, bakterielle und mykotische Hauterkrankungen. Nebenwirkungen: Es kann zU systemischen Effekten infolge Resorption nach topischer Anwendung von corticoidhaltigen Arzneimitteln kommen. Häufig: a. d. Appl.stelle Milch, Lösung: Brennen; Salbe, Creme: Brennen u. Juckreiz; Fettsalbe: Brennen U. Follikulitis; Gelegentl.: Milch: Ekzeme, Exfoliation der Haut, Hautfissuren, a. d. Appl.stelle Schmerzen, Blaschen, Pruritus, Papeln, Erosion; Salbe: Hautatrophie, Ekchymose, Impetigo, fettige Haut, a. d. Appl.stelle Erythem, Trockenheit, Bläschen, Reizung, Ekzem, periph. Odem; Fettsalbe: Hautfissuren, Teleangiektasien, a. d. Appl.stelle Pusteln, Bläschen, Pruritus, Schmerzen, Erythem, Papeln; Creme: Uberempfindlichkeit gegen den Wirkstoff; a. d. Appl.stelle Hauttrockenheit, Erythem, Bläschen, Follikulitis, Ausschlag, Parästhesie; Lösung: a. d. Appl.stelle Pruritus, Schmerzen, Follikulitis, Wärme, Trockenheit, Reizung, Ekzem; Seborrhoe d. Kopfhaut, Haarausfall. Selten: Creme: Pilzinfekt. der Haut, Pyodermitis, Hautfissuren, Teleangiektasien, Hautatrophie, Akne a. d. Appl.stelle Cellulitis, Ödem, Hautreizungen. Häufigkeit nicht bekannt: Striae, Hypertrichose, periorale Dermatitis, Hautverfärbung, allerg. Hautreaktionen; Milch zusätzl.: a. d. Appl.stelle Hautatrophie, follikulitis, Trockenheit U. Erythem; Teleangiektasien, Akne; fettsalbe zusätzl.: Überempfindlichkeit gegen den Wirkstoff, Akne, Hautatrophie; Salbe zusätzl:: Uberempfindlichkeit gegen den Wirkstoff, Akne, Teleangiektasien; a. d. Appl.stelle Follikulitis; Lösung zusätzl.: a.d. Appl.stelle Bläschen, Erythem; Akne, Teleangiektasien, Hautatrophie. Packungen: Milch: Tube mit $20 \mathrm{~g}, 50 \mathrm{~g}$ Appl.stelle Follikulitis; Losung zusatzl.: a.d. Appl.stelle Blaschen, Erythem; Akne, Teleangiektasien, Hautatrophie. Packungen: Milch: Tube mit $20 \mathrm{~g}, 50 \mathrm{~g}$,
$100 \mathrm{~g}$. Salbe/Fettsalbe/Creme: Tube mit $15 \mathrm{~g}, 25 \mathrm{~g}, 50 \mathrm{~g}, 100 \mathrm{~g}$. Lösung: Flasche mit $30 \mathrm{ml}, 50 \mathrm{ml}$. Dosierung: 1-mal täglich dünn auftragen. Warnhinweise: Creme: Cetylstearylalkohol und Butylhydroxytoluol können lokale Hautreizungen hervorrufen. Butylhydroxytoluol kann auch Reizungen der Augen Creme: Cetylstearylalkohol und Butylhydroxytoluol können lokale Hautreizungen hervorrufen. Butylhydroxytoluol kann auch Reizungen der Augen
und Schleimhäute hervorrufen. Verschreibungspflichtig. Pharmazeutischer Unternehmer: Jenapharm GmbH \& Co KG, Otto-Schott-Straße 15, 07745 Jena, Stand: Milch FI/13, 05/2014; Salbe FI/7, 02/2014; Fettsalbe FI/7, 02/2014; Creme FI/7, 02/2014; Lösung FI/12, 11/2013 
Abb. 6. Zerkariendermatitis: Dermatitis mit zahlreichen erodierten Papulovesikeln.

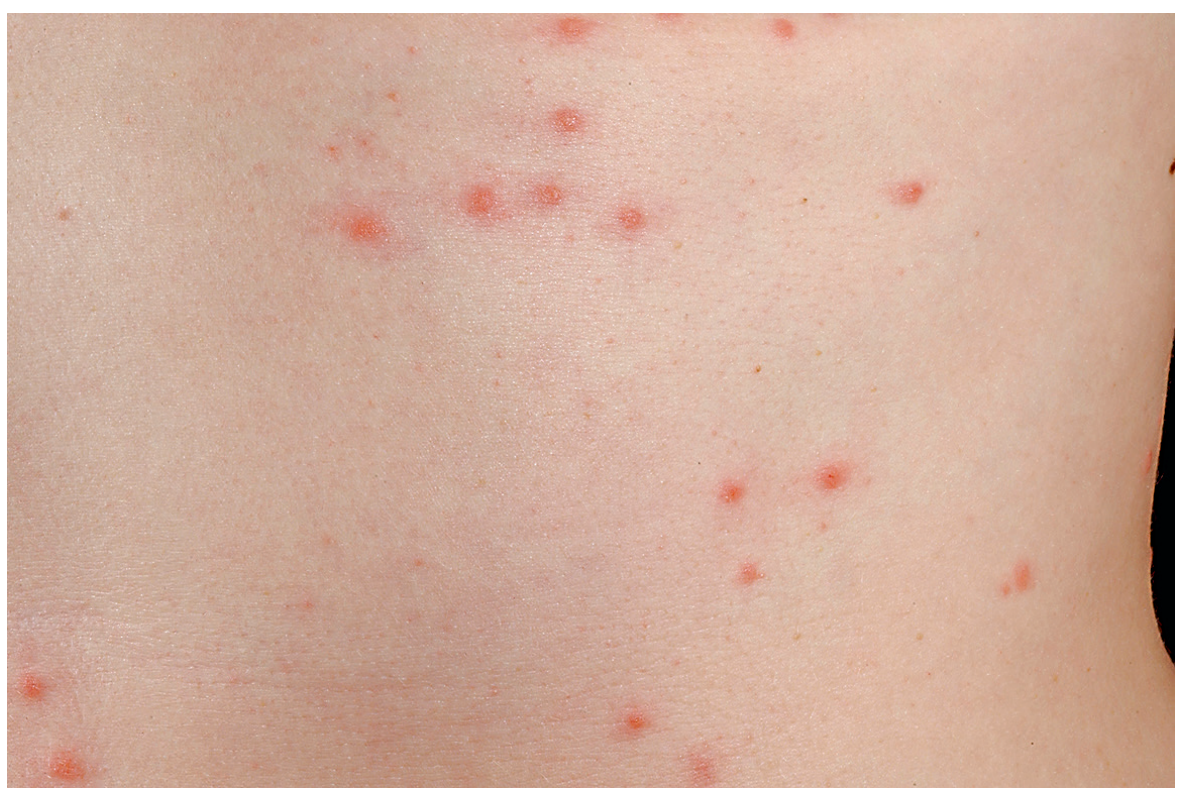

Die Krankheit wird von vielen Ärzten nicht erkannt und fehldiagnostiziert [64]. Im Durchschnitt vergeht 1 Jahr, bevor die richtige Diagnose gestellt wird, wobei die Kinder in dieser Zeit meist antimykotische Medikamente erhalten [63]. Es gibt Hinweise darauf, dass Lichen sclerosus nach der Pubertät nicht abklingt [64]. Für 36 Mädchen vor der Menarche (mittleres Alter bei Diagnose: 7 Jahre), bei denen zwischen 1989 und 2010 Lichen sclerosus diagnostiziert wurde, wurden eine retrospektive Analyse der Krankenblätter und ein Nachsorge-Telefoninterview [65] durchgeführt. Die mittlere Dauer der Nachbeobachtung betrug 5,3 Jahre. Bei 33 Mädchen kam es bei Anwendung topischer Kortikosteroide innerhalb von durchschnittlich 14 Wochen (Bereich: 2 Wochen bis 2 Jahre) zu einer Besserung der Symptome. Während 83\% eine Remission nach der Erstbehandlung zeigten, erlebten $16 \mathrm{~Pa}$ tientinnen Rückfälle, die eine weitere Therapie erforderlich machten. Es empfiehlt sich, die Kinder mindestens bis zur Pubertät zu beobachten [66]. Lichen sclerosus sollte mit starken Kortikosteroiden oder Calcineurin-Inhibitoren behandelt werden [63]. $\mathrm{Zu}$ deren Vorteilen gehört die Linderung von Pruritus, Schmerz und Entzündungen [67]. Folgeerkrankungen wie Vulvastenose oder Plattenepithelkarzinom sind sehr selten im Kindesalter [63].

\section{Bullöse und Urtikaria-Dermatosen}

\section{Epidermolysis bullosa}

Epidermolysis bullosa (EB), eine heterogene Gruppe bullöser, durch Traumata ausgelöster Dermatosen, ist mit einem starken Pruritus assoziiert. Es gibt die erworbene und die erbliche Form, wobei Letztere in die vernarbende (dystrophische) und die nicht vernarbende Form (Unterscheidung zwischen EB intraepidermales und EB junctionalis) unterteilt ist. Diese können auf Grundlage der klinischen Merkmale, des Vererbungsmodus, der Histopathologie und molekularbiologischer Untersuchungen definiert werden. Die Therapie ist symptombezogen und hat das Ziel, Infektionen zu verhindern.
Einige Publikationen konzentrieren sich auf den Pruritus bei Patienten mit Epidermolysis bullosa. Die wichtigsten Botschaften auf Basis klinischer Studien sind Folgende [68]:

- Pruritus ist ein verbreitetes und belastendes Symptom bei Patienten mit Epidermolysis bullosa.

- Die durchschnittliche tägliche Häufigkeit des Pruritus steigt mit der von den Patienten selbst angegebenen Schwere der Epidermolysis bullosa.

- Der Pruritus tritt am häufigsten während der Schlafenszeit auf und stört den Schlaf.

- Zu den Faktoren, die den Pruritus verschlimmern, gehören heilende Wunden, trockene Haut, infizierte Wunden, Stress, Hitze, Trockenheit und Feuchtigkeit.

Das Management von Patienten mit Epidermolysis bullosa erfordert eine interdisziplinäre Zusammenarbeit (Augenärzte, Gastroenterologen, Hals-Nasen-Ohren (HNO)-Ärzte, plastische Chirurgen, Zahnärzte und Physiotherapeuten), um betroffene extrakutane Bereiche zu behandeln.

$\mathrm{Zu}$ den Präventionsmaßnahmen gehören die Vermeidung von Traumata und Infektionen der Haut und Schleimhäute sowie das Tragen geeigneter Kleidung und Anlegen von Wundverbänden, die eine ausreichende Hydratation und ein schmerzfreies Wechseln der Bandagen ermöglichen. Darüber hinaus sollten Schulungsprogramme in das therapeutische Management aufgenommen werden, um besser mit dem Pruritus umgehen zu können [68].

Kürzlich konnte gezeigt werden, dass der Einsatz von Salzwasserbädern zu einer signifikanten Verbesserung der Lebensqualität führen kann [69]. Nachdem Patienten mit den Salzwasserbädern begonnen hatten, gaben sie mithilfe eines standardisierten Fragebogens eine signifikante Verringerung von Schmerzen, Schmerzmittelgebrauch, Hautgeruch und Hautabschuppung an. Eine weitere Studie [70] konzentrierte sich auf die Bewertung verschiedener Behandlungsmodalitäten anhand eines Fragebogens. Die Fragen bezogen sich auf Badeprodukte, Feuchtigkeitscremes, to- 
Tab. 3. Juckreiz im Windelbereich und seine Ursachen (Auswahl)

\begin{tabular}{|c|c|c|}
\hline Krankheiten & Klinisches Muster & Alter bei Krankheitsbeginn \\
\hline \multicolumn{3}{|l|}{ Entzündliche Krankheiten } \\
\hline Psoriasis & $\begin{array}{l}\text { Erythem und Schuppung auf der Kopfhaut und } \\
\text { in den großen Körperfalten }\end{array}$ & jedes Alter \\
\hline Atopische Dermatitis & $\begin{array}{l}\text { Ekzeme auf Kopfhaut, Gesicht, Extremitäten, } \\
\text { Atopie; Windelbereich oft frei von Läsionen; im } \\
\text { höheren Kindesalter sind Ekzeme im Genito- } \\
\text { analbereich möglich }\end{array}$ & vor allem im 1. Lebensjahr \\
\hline \multicolumn{3}{|l|}{ Autoimmunerkrankungen } \\
\hline Lichen sclerosus (genitalis) & $\begin{array}{l}\text { «Achterfigur» bei Mädchen mit porzellanweißen } \\
\text { Papeln und Plaques }\end{array}$ & $\begin{array}{l}\text { Mädchen vor der Menarche, } \\
\text { weniger häufig bei Jungen } \\
\text { im Vorschulalter; mit Phimose } \\
\text { assoziiert }\end{array}$ \\
\hline Lineare IgA-Dermatose & $\begin{array}{l}\text { blasenbildende ringförmige oder rosettenartige } \\
\text { Läsionen an Beinen, Füßen, Rumpf }\end{array}$ & Vorschulkinder \\
\hline Epidermolysis bullosa & $\begin{array}{l}\text { abhängig von der Form: Erosionen, Blasen, } \\
\text { Narben; durch Trauma ausgelöst }\end{array}$ & $\begin{array}{l}\text { abhängig von der Form: bei } \\
\text { der Geburt oder im frühen } \\
\text { Kindesalter }\end{array}$ \\
\hline
\end{tabular}

Infektionen und Infestationen

Candidiasis Intertrigo und Satelliten-Papulopusteln

Kinder, Mädchen vor der Menarche und im Jugendalter (Vulvovaginitis)

\begin{tabular}{lll}
\hline $\begin{array}{l}\text { Perianale Streptokokken-Dermatitis } \\
\text { (oder Staphylokokken- } \\
\text { Dermatitis) }\end{array}$ & $\begin{array}{l}\text { perianales Erythem mit feuchter Oberfläche, } \\
\text { perianaler und rektaler Juckreiz, schmerzhafte } \\
\text { Defäkation }\end{array}$ & vorpubertäre Kinder \\
\hline Skabies & $\begin{array}{l}\text { Gänge, verbreitetes Ekzem, einschließlich Gesicht } \\
\text { und Kopf, Pusteln an Fußsohlen }\end{array}$ & jederzeit \\
\hline $\begin{array}{l}\text { Stoffwechselstörungen } \\
\text { Akrodermatitis enteropathica }\end{array}$ & $\begin{array}{l}\text { Trias aus ekzematösen Läsionen (periorifiziell, } \\
\text { Finger und Zehen), Alopezie und Durchfall }\end{array}$ & $\begin{array}{l}\text { 2 Wochen nach dem } \\
\text { Abstillen }\end{array}$ \\
\hline Neoplasie & $\begin{array}{l}\text { bräunliche Papeln, Papulovesikel, impetigoartige } \\
\text { Langerhans-Zell-Histiozytose } \\
\text { rhoischen Läsionen auf der Kopfhaut }\end{array}$ & $\begin{array}{l}\text { bei der Geburt oder in den } \\
\text { ersten } 2 \text { Jahren }\end{array}$ \\
\hline
\end{tabular}

pische Produkte, orale Medikamente, Verbände und alternative Therapien. Die am häufigsten verwendeten Pruritus-Behandlungsoptionen waren nach Häufigkeit Fettsalben, Lotionen, Cremes und orales Hydroxyzin (39,0\%). Am wirksamsten waren topische Kortikosteroide, orales Hydroxyzin, topisches Diphenhydramin und Externa, die ätherische Öle enthalten (Menthol, Kampfer, Eukalyptus). Es wurde festgestellt, dass systemische Opioide, haftende Bandagen und Bleichbäder den Pruritus leicht erhöhten.

\section{Mastozytose}

Eine Mastozytose (Anhäufung von Mastzellen) tritt bei Kindern überwiegend in Form von Mastozytomen und Urticaria pigmentosa auf (Abb. 7). Die anderen Formen (bullosa, diffusa), bei denen auch extrakutane Organsysteme wie der Gastrointestinaltrakt, das Skelett und das Knochenmark betroffen sind, kommen im Vergleich zu Erwachsenen selten vor [71]. Die Diagnose wird anhand der Morphologie und eines positiven Darier-Zeichens gestellt. Besonders Kleinkinder neigen dazu, durch die mechanische Reizung des Darier-Zeichens starke Reaktionen zu entwickeln, wodurch sich nicht nur Quaddeln und Blasen bilden können, sondern auch Flush (Erröten) auftreten kann. Neben dem mechanischen Auslöser können extreme Temperaturen (Hitze, Kälte), körperliche Betätigung, Nahrungsmittel, Medikamente und Insektengifte zur Mastzelldegranulation führen. Verglichen mit Erwachsenen ergibt sich für Kinder eine sehr gute Prognose, und es kommt sehr häufig zur Remission. Es erfolgt eine symptombezogene Therapie, bei der auslösende Faktoren vermieden und H-1-Antihistaminika bei Bedarf verabreicht werden.

Es gibt ein hilfreiches Scoring-System (SCORMA), das mit dem Tryptase-Spiegel im Serum korreliert und die Ausdehnung sowie die Intensität der Läsionen und subjektive Symptome wie Pruritus mit einbezieht $[72,73]$. 


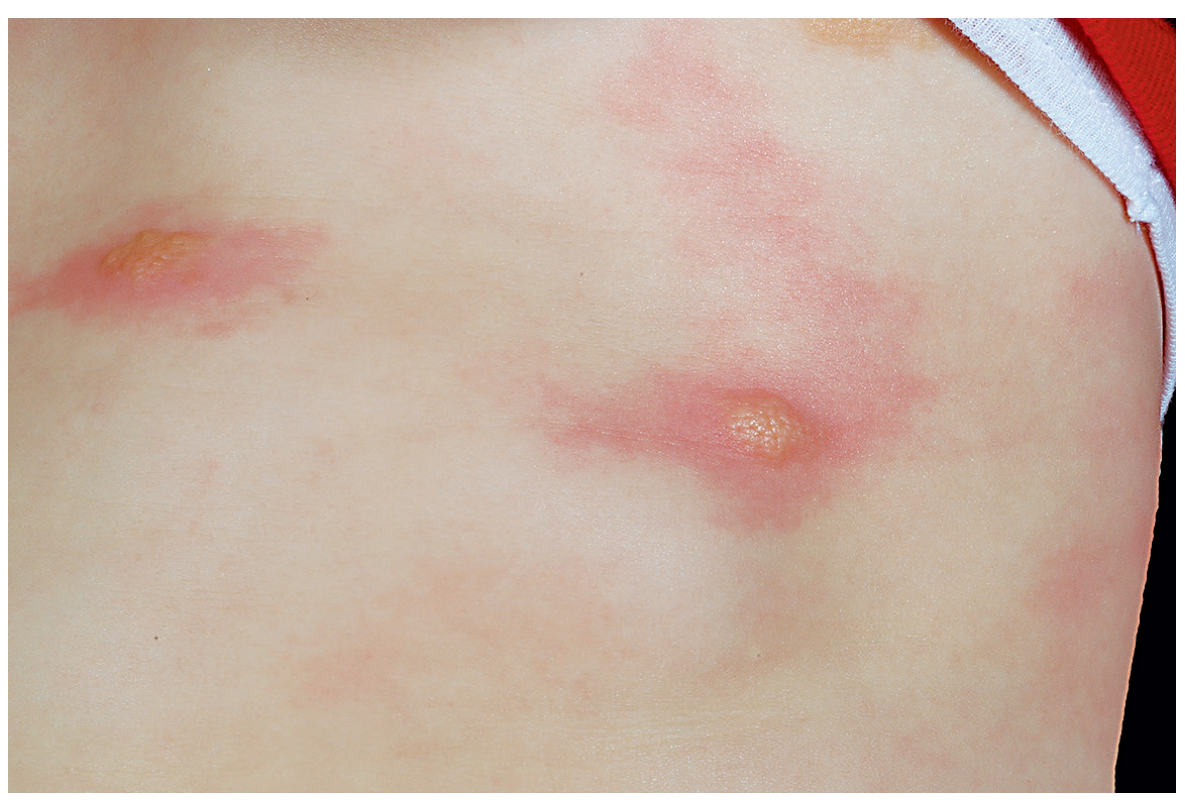

\section{Urtikaria}

Urtikaria wird definiert als plötzliches Auftreten stark juckender Quaddeln, die meist einige Stunden bis einen Tag andauern. Je nach Dauer und Ätiologie wird Urtikaria in die folgenden Typen klassifiziert: spontane akute Urtikaria, spontane chronische Urtikaria, physikalische Urtikaria und weitere Urtikaria-Typen [74, 75]. Diese Typen erfordern jeweils ein unterschiedliches Management.

Die Erkrankung ist im Kindesalter sehr verbreitet und tritt sehr häufig als akute Urtikaria auf (Dauer weniger als 6 Wochen), die häufig mit Virusinfektionen assoziiert sind. In einer Studie an 54 Kindern mit Urtikaria stellten Sackesen et al. [76] fest, dass 68,5\% an der Akutform litten. Bei etwa der Hälfte dieser Patienten war die Ursache eine Infektion (48,6\%), während physikalische Faktoren die Hauptursache in der Gruppe der chronischen Urtikaria darstellten (52,94\%). Neben einer detaillierten Anamnese empfahlen die Autoren die Diagnostik hinsichtlich möglicher Infektionserreger. Die Ursache einer chronischen Urtikaria bei Kindern konnte zu 20-50\% identifiziert werden [77]. Bei 30-47\% dieser Kinder hängt die Urtikaria jedoch mit einer autoreaktiven Form zusammen (bestätigt durch einen positiven «autologen Serumhauttest»), die durch Immunglobulin-G (IgG)-Antikörper gegen den hochaffinen IgE-Rezeptor hervorgerufen wird (FceRI $\alpha$ ) [78].

Antihistaminika der zweiten Generation bilden die Grundlage der pharmakologischen Behandlung [75, 77]. Omalizumab wird in refraktären Fällen empfohlen, besonders bei Kindern mit autoreaktiver chronischer Urtikaria [79].

\section{Tumoren als Ursache lokalisierten Juckreizes}

Lokalisierter Pruritus kann durch Tumoren des Nervensystems bedingt sein. Soltani-Arabshahi et al. [80] empfehlen eine neurologische Untersuchung und eine entsprechende Bildgebung bei Kindern mit persistierendem lokalisiertem Pruritus, wenn keine anderen Ursachen von Pruritus vorliegen. Die Ergebnisse der Literaturrecherche der Autoren zeigten, dass Astrozytome und Gliome die häufigsten intramedullären Neoplasmen darstellen, die mit Pruritus in der pädiatrischen Patientengruppe zusammenhängen. Ferner fanden die Autoren 6 Artikel über Kinder, die aufgrund von Hirnstamm- oder Rückenmarkstumoren an persistierendem lokalisiertem Pruritus an Kopf und Hals oder an den oberen Extremitäten litten [80]. 5 dieser Kinder wiesen Caféau-lait-Flecken in Verbindung mit einer Neurofibromatose auf. Im folgenden Abschnitt geht es schwerpunktmäßig um die Neurofibromatose Typ 1, bei der juckende dermale Neurofibrome auftreten, die vermutlich durch das Vorhandensein von Mastzellen bedingt sind. Diese Tumoren treten nicht vor der Pubertät auf.

\section{Neurofibromatose Typ 1 (Morbus Recklinghausen)}

Die Neurofibromatose Typ 1 (NF1) ist eine autosomal-dominante Erkrankung aufgrund einer Mutation des Tumorsuppressorgens NF1 (17q11.2), das für das Ras-GTPase-aktivierende Protein Neurofibromin kodiert, das im ganzen Nervensystem exprimiert wird [81]. Die Inaktivierung des Tumorsuppressorgens ist mit einem hohen Risiko für bestimmte Tumortypen verbunden, die aus der embryonalen Neuralleiste hervorgehen. Die NF1-Diagnose wird gestellt, wenn mindestens 2 von 7 klinischen Kriterien vorliegen: $\geq 6$ Café-au-lait-Flecken $>0,5 \mathrm{~cm}$, Pigmentflecken in Achselhöhlen und Leistengegend, $\geq 2$ Neurofibrome oder 1 plexiformes Neurofibrom, $\geq 2$ Lisch-Knötchen, optisches Gliom, eine charakteristische Knochenläsion (Pseudarthrose oder sphenoidale Dysplasie) oder ein Verwandter ersten Grades mit NF1 [82]. Etwa jeder fünfte Patient leidet an Pruritus [83]. Brenaut et al. [81] evaluierten die Eigenschaften des Pruritus bei 40 Patienten (Erwachsenen) mit NF1 anhand eines Fragebogens. Zu den wichtigsten Erkenntnissen gehörte, dass ein Drittel der Patienten täglich an Pruritus leidet, wobei der Juckreiz eher abends auftritt; nur bei 5 Patienten wurde der Pruritus behandelt (mit Emollientien, Antihistaminika); die Intensität des Pruritus wurde anhand einer 
Tab. 4. Topische antipruritische Therapie bei Kindern (basierend auf Lucas et al. [1] und Eichenfield [85])

\begin{tabular}{|c|c|c|c|}
\hline $\begin{array}{l}\text { Wirkstoff/zugelassen ab einem } \\
\text { Alter von: }\end{array}$ & Dosierung & Indikation (Auswahl) & Nebenwirkungen/Besonderheiten \\
\hline \multirow[t]{3}{*}{$\begin{array}{l}\text { Urea pura 5-10\%/nicht für } \\
\text { Kleinkinder }\end{array}$} & \multirow[t]{3}{*}{ 2-3-mal täglich } & ekzematöse Erkrankungen & $\begin{array}{l}\text { im Kleinkindalter und bei akuten } \\
\text { Läsionen }\end{array}$ \\
\hline & & Ichthyosen & «stechender Effekt» \\
\hline & & Psoriasis vulgaris & \\
\hline
\end{tabular}

Calcineurin-Inhibitoren/zugelassen

ab 2 Jahren

\begin{tabular}{|c|c|c|c|}
\hline Pimecrolimus & 2-mal täglich & $\begin{array}{l}\text { leichte bis mäßige atopische } \\
\text { Dermatitis }\end{array}$ & $\begin{array}{l}\text { vorübergehendes Brennen an der } \\
\text { Anwendungsstelle/Sonnenschutz }\end{array}$ \\
\hline \multirow[t]{2}{*}{ Tacrolimus } & 2-mal täglich & $\begin{array}{l}\text { mäßige bis schwere atopische } \\
\text { Dermatitis }\end{array}$ & \\
\hline & & $\begin{array}{l}\text { Off-Label-Verwendung beider } \\
\text { Medikamente bei Lichen } \\
\text { sclerosus }\end{array}$ & \\
\hline
\end{tabular}

Kortikosteroide

Methylprednisolonaceponat/ 1-mal (bei Bedarf 2-mal) täglich

Exazerbation atopischer

Dermatitis, andere

bei ordnungsgemäßer Verwendung zugelassen ab 3 Jahren entzündliche Dermatosen keine Nebenwirkungen/keine intertriginöse Anwendung

Mometasonfuroat/zugelassen 1-mal (bei Bedarf 2-mal) täglich

Exazerbation atopischer Dermatitis, andere bei ordnungsgemäßer Anwendung keine Nebenwirkungen entzündliche Dermatosen keine intertriginöse Anwendung

Prednicarbat/im Kleinkindalter nur 1-mal (bei Bedarf 2-mal) täglich nach sorgfältiger Abwägung

Exazerbation atopischer Dermatitis, andere entzündliche Dermatosen bei ordnungsgemäßer Anwendung keine Nebenwirkungen/ keine intertriginöse Anwendung

Antiparasitäre Medikamente

Benzylbenzoat 10\%/nicht für

Frühgeborene

3 Tage lang 1-mal täglich, Skabies am 4. Tag abwaschen
Reizung der Haut und Schleimhäute/ Sensibilisierung möglich, «GaspingSyndrom» bei Frühgeborenen

geringere Wirksamkeit als andere Skabizide

Besuch von Kindergarten/Schule 1 Tag nach einer vorschriftsmäßig ausgeführten Behandlung

beschränkung

Skabies: 1-mal täglich 8 Stunden lang; Pediculosis capitis: 1-mal nach dem Haarewaschen für 30-45 min
Skabies, Pediculosis capitis

esuch von Kindergarten/Schule 1 Tag nach einer vorschriftsmäßig ausgeführten Behandlung visuellen Analogskala (0-10) beurteilt: extrem (6,7/10), leicht (1/10), woraus sich eine mittlere Intensität des Pruritus von 3,8/10 ergab. Bei 23,5\% bzw. 17,6\% der Patienten traten auch Schmerzen bzw. Hitzeempfindungen auf. Bei der Hälfte der Patienten war der Pruritus auf die Neurofibrome begrenzt.

In einer großen Kohorte erwachsener und pädiatrischer Patienten war das Vorhandensein fazialer plexiformer Neurofibrome und von Pruritus bei Kindern in einer univariaten Analyse in signifikanter Weise mit Mortalität assoziiert [83]. Die Autoren empfahlen eine engmaschige Nachbeobachtung für Patienten mit subkutanen Neurofibromen.

\section{Systemische Erkrankungen und Arzneimittel}

Pruritus in Verbindung mit systemischen Erkrankungen oder unerwünschten Ereignissen bei Arzneimitteln ist bei Kindern seltener als bei Erwachsenen. Dennoch tritt er auf und betrifft dieselben Organsysteme wie bei den Erwachsenen. Der Unterschied besteht darin, dass es sich bei diesen Erkrankungen um typische Kinderkrankheiten handelt, zu denen hauptsächlich Genodermatosen gehören [84]. Weisshaar et al. [84] geben einen Überblick über die systemischen Erkrankungen im Kindesalter, die mit Pruritus einhergehen. 
Tab. 5. Systemische antipruritische Therapie bei Kindern (basierend auf Lucas et al. [1], Cuvillo [86] und Fitzsimons [87])

\begin{tabular}{|c|c|c|c|}
\hline $\begin{array}{l}\text { Wirkstoff/ zugelassen ab einem } \\
\text { Alter von: }\end{array}$ & Dosierung & Indikation (Auswahl) & $\begin{array}{l}\text { (Mögliche) Nebenwirkungen } \\
\text { /Besonderheiten }\end{array}$ \\
\hline \multicolumn{4}{|l|}{ H1-Antihistaminika } \\
\hline Acrivastin/12-18 Jahre & 12-18 Jahre: $3 \times$ täglich 8 mg & Urtikaria & \multirow{6}{*}{$\begin{array}{l}\text { häufigste Nebenwirkungen der } \\
\text { aufgeführten Antihistaminika (die } \\
\text { alle der 2. Generation angehören): } \\
\text { Müdigkeit, Schwindel, trockener } \\
\text { Mund, Kopfschmerz/keine kardio- } \\
\text { vaskulären Nebenwirkungen }\end{array}$} \\
\hline Ceterizin/1-18 Jahre & $\begin{array}{l}\text { 1-2 Jahre: } 2 \times \text { täglich } 250 \mu / \mathrm{kg} \\
\text { 2-6 Jahre: } 2 \times \text { täglich 2,5 mg/kg } \\
\text { 6-12 Jahre: } 2 \times \text { täglich } 5 \text { mg/kg } \\
\text { 12-18 Jahre: } 1 \times \text { täglich } 10 \text { mg/kg }\end{array}$ & $\begin{array}{l}\text { Urtikaria, atopische Dermatitis, } \\
\text { infantile Akropustulose }\end{array}$ & \\
\hline Desloratidin/1-18 Jahre & $\begin{array}{l}\text { 1-6 Jahre: } 1 \times \text { täglich 1,25 mg } \\
\text { 6-12 Jahre: } 1 \times \text { täglich 2,5 mg } \\
\text { 12-18 Jahre: } 1 \times \text { täglich } 5 \text { mg }\end{array}$ & Urtikaria & \\
\hline Fexofenadin/6-18 Jahre & $\begin{array}{l}\text { 6-12 Jahre: } 2 \times \text { täglich } 30 \text { mg } \\
\text { 6-18 Jahre: } 1 \text { × täglich } 120 \text { mg }\end{array}$ & Urtikaria & \\
\hline $\begin{array}{l}\text { Levozeterizin/ } \\
\text { Flüssigkeit: 2-18 Jahre } \\
\text { Tabletten: 6-18 Jahre }\end{array}$ & $\begin{array}{l}\text { 2-6 Jahre: } 2 \times \text { täglich 1,25 mg } \\
\text { 6-18 Jahre: } 1 \times \text { täglich } 5 \text { mg }\end{array}$ & Urtikaria & \\
\hline Loratidin/2-18 Jahre & $\begin{array}{l}\text { 2-12 Jahre: } \\
\text { < } 30 \text { kg: } 1 \times \text { täglich } 5 \text { mg } \\
\text { > } 30 \text { mg: } 1 \times \text { täglich } 10 \text { mg } \\
\text { 12-18 Jahre: } 1 \times \text { täglich } 10 \text { mg }\end{array}$ & Urtikaria & \\
\hline $\begin{array}{l}\text { Ciclosporin (Mikroemulsion)/ } \\
\text { ab } 16 \text { Jahren }\end{array}$ & $3-5 \mathrm{mg} / \mathrm{kg} / \mathrm{Tag}$ & $\begin{array}{l}\text { atopische Dermatitis, refraktäre } \\
\text { Urtikaria }\end{array}$ & $\begin{array}{l}\text { am häufigsten bei Kindern: } \\
\text { gastrointestinale Symptome und } \\
\text { Kopfschmerz; daneben: Hypertonie, } \\
\text { Nephrotoxizität, Gingivahyperplasie, } \\
\text { Hypertrichose, Myalgie, Hyperli- } \\
\text { pidämie/Wechselwirkungen mit } \\
\text { anderen Arzneimitteln }\end{array}$ \\
\hline $\begin{array}{l}\text { Dapson (Sulfone)/nicht für Kinder } \\
\text { zugelassen; Empfehlung: nicht im } \\
\text { frühen Kindesalter verwenden }\end{array}$ & 0,5 mg/kg/Tag & $\begin{array}{l}\text { infantile Akropustulose, die } \\
\text { nicht auf die Standardtherapie } \\
\text { mit topischen Kortikosteroiden } \\
\text { und H1-Antihistaminika } \\
\text { anspricht; Autoimmun- } \\
\text { erkrankungen }\end{array}$ & $\begin{array}{l}\text { Kopfschmerz, hämolytische Anä- } \\
\text { mie, Methämoglobinämie, Übelkeit, } \\
\text { Überempfindlichkeitsreaktionen/ } \\
\text { Baseline-Blutbilder } \\
\text { und Glukose-6-phosphat-Dehydro- } \\
\text { genase (G6PD) }\end{array}$ \\
\hline $\begin{array}{l}\text { Ivermectin (antiparasitäres } \\
\text { Medikament) }\end{array}$ & $\begin{array}{l}\text { Einzeldosis von } 150-200 \mu \mathrm{g} / \mathrm{kg} \text {; } \\
\text { nach 8-10 Tagen wiederholen }\end{array}$ & $\begin{array}{l}\text { im Falle refraktärer Parasito- } \\
\text { sen (z.B. Pediculosis capitis, } \\
\text { Skabies) }\end{array}$ & $\begin{array}{l}\text { Fieber, Pruritus, Hautausschlag; } \\
\text { Wechselwirkungen mit anderen } \\
\text { Arzneimitteln }\end{array}$ \\
\hline
\end{tabular}

Die folgenden Fragen sind hilfreich, um die korrekte Diagnose zu stellen:

- Findet sich in der Anamnese eine systemische Erkrankung?

- Sind genetische Erkrankungen und/oder Krankheiten in der Familie bekannt, die mit Lebererkrankungen wie Gallengangatresie oder -hypoplasie, dem Alagille-Syndrom (Fehlen interlobulärer Gallengänge), choledochalen Zysten und einem familiären Hyperbilirubinämie-Syndrom einhergehen, wie z.B. die BylerKrankheit (intrahepatische Cholestase) und der hämolytische Ikterus [84]?

- Leidet das Kind an einer polyzystischen Nierenkrankheit?

- Liegt der Serum-Eisenspiegel im normalen Bereich?

- Hat das Kind eine Lymphadenopathie (z.B. Morbus Hodgkin)?
- Ist das Blutbild normal (im Hinblick auf Leukämie oder Polycythaemia vera)?

- Sind Anzeichen von AIDS vorhanden wie wiederkehrende Virusinfektionen (vor allem Molluscum contagiosum), bakterielle oder mykotische Infektionen oder seborrhoische Dermatitis?

- Hat das Kind Medikamente eingenommen, insbesondere Antibiotika, Antikonvulsiva, nichtsteroidale Antiphlogistika oder Morphin?

Die Tabellen 4 und 5 geben einen Überblick über die topischen und systemischen Medikationen, die bei Kindern am häufigsten verwendet werden.

\section{Literatur}

Literatur online unter $w w w$.karger.com/?doi $=454965$ 